\title{
EL BRONCE CELTIBÉRICO DE EL CASTILLO DE RABANERA (VENTOSA, SAN PEDRO MANRIQUE, SORIA)
}

\section{THE CELTIBERIAN BRONZE FROM EL CASTILLO DE RABANERA (VENTOSA, SAN PEDRO MANRIQUE, SORIA)}

\author{
Eduardo Alfaro PeñA* \\ Proyecto Idoubeda oros \\ Joan Ferrer I Jané \\ Grup Littera, Universitat de Barcelona \\ † Joaquín Gómez-Pantoja Fernández-Salguero \\ Universidad de Alcalá de Henares
}

\begin{abstract}
RESUMEN. En este estudio se presenta la edición de un fragmento de lámina de bronce hallado irregularmente en el yacimiento celtibérico de El Castillo de Rabanera (San Pedro Manrique, Soria) que contiene una inscripción en lengua celtibérica y escritura epicórica occidental en el que se identifican algunos de los elementos léxicos más característicos de este tipo de soportes, como auzar[es y ueizui. El elemento más problemático de este nuevo texto es el símbolo al principio de la tercera línea, que podría estar reproduciendo la misma casuística que la ya documentada en el bronce Res. Los principales paralelos tanto formales como de contenido de esta inscripción son los bronces de Cortono, 'Res', Luzaga y Torrijo. Otro elemento destacado de la inscripción es riounu, que probablemente identifica alguna de las ciudades o personas intervinientes en el documento, quizás a la propia ciudad emisora del documento.
\end{abstract}

PALABRAS CLAVE. Lengua celtibérica, inscripción celtibérica, Soria, lámina de bronce.

ABSTRACT. This paper deals with a fragment of bronze plaque found irregularly in the Celtiberian site of El Castillo de Rabanera (San Pedro Manrique, Soria) that contains an inscription in Celtiberian language and western epichoric writing. The main formal and content parallels of this inscription are the bronzes of Cortono, 'Res', Luzaga and Torrijo as the text contains some of the most characteristic elements of these objects, such as auzar[es] and ueizui. Another noteworthy element of the inscription is riounu, which probably identifies some of the cities or people involved in the document, perhaps the city that issued the document. Nevertheless, the most problematic element of this text is the symbol at the beginning of the third line, which reminds the solar symbol in the bronze 'Res'.

KEYWORDS. Celtiberian language, Celtiberian inscription, Soria, bronze sheet.

\footnotetext{
* Correspondencia a / Correspondence to: Eduardo Alfaro Peña, C/ Las Peñas, 18 (42176 Santa Cruz de Yanguas, Soria) - idoubeda@gmail.com https://orcid.org/0000-0002-8300-2348.

Cómo citar / How to cite: Alfaro Peña, Eduardo; Ferrer i Jané, Joan; † Gómez-Pantoja Fernández-Salguero, Joaquín (2021), «El bronce celtibérico de El Castillo de Rabanera (Ventosa, San Pedro Manrique, Soria)», Veleia, 38, 111-139. (https://doi.org/10.1387/veleia.21987).

Recibido: 6 septiembre 2020; aceptado: 4 octubre 2020.
}

ISSN 0213-2095 - eISSN 2444-3565 / (C) 2021 UPV/EHU 


\section{EDUARDO ALFARO PEÑA, JOAN FERRER I JANÉ, † JOAQUÍN GÓMEZ-PANTOJA FDEZ.-SALGUERO}

\section{INTRODUCCIÓN ${ }^{1}$}

Este artículo nace huérfano de una de sus firmas, la de Joaquín Gómez-Pantoja, el cordón que ha unido y motivado esta colaboración a tres. Y decimos huérfano porque fue Joaquín el que decidió sacar del rincón de los recelos a esta pieza de bronce localizada fuera de contexto estratigráfico en las Tierras Altas de Soria. Más allá de las dudas que pudiera generar tenía claro que era necesario darla a conocer y así lo hacemos. Joaquín nos dejó el pasado agosto; en lo inmediato y para este trabajo hemos echado en falta sus observaciones y su buena pluma, en el futuro al maestro y al amigo.

Centrados ya en la pieza, la información que se nos ha aportado asegura que fue localizada en El Castillo de Rabanera por el vecino de Ventosa de San Pedro que la conserva; fue enseñada a uno de nosotros (EAP) al que se permitió y facilitó su documentación gráfica.

\section{El yacimiento}

Es Rabanera un despoblado moderno que remonta su origen a los tiempos celtibéricos de los que quedan interesantes restos de estructuras defensivas. Tanto a estas como al espacio que cierran se denomina El Castillo, que guarda las características habituales de las unidades básicas del poblamiento comarcal de la Segunda Edad del Hierro: un pequeño poblado encastillado con defensa natural en tres de sus flancos, levantando en el cuarto un poderoso bastión proyectado en muralla hasta cerrarlo, sistema defensivo básico que se complementa con foso previo en el que se dejó un pasillo de acceso. Extramuros algunos materiales cerámicos aseguran su ocupación en época altoimperial que las cerámicas a torneta amplían hasta la Alta Edad Media. En la zona más acogedora de la vaguada oriental extramuros se localizan los derrumbes de piedras que señalan el emplazamiento del despoblado medieval/moderno en el que aún se mantienen en pie algunos muros de la iglesia, hoy ruinas de la ermita de Rabanera, de fundamentos románicos. Intramuros, con propiedad en El Castillo, además de las estructuras y los materiales de cronología celtibérica, hay algunas evidencias de ocupación imperial, especialmente de la tardía (Alfaro 2005, 128-130).

Del momento que cronológicamente interesa, el periodo más avanzado de la Segunda Edad del Hierro, se conocen algunos materiales metálicos, como la tabula, recogidos y custodiados por vecinos del pueblo: un fragmento de fíbula ancoriforme, una fíbula de caballito del tipo E2 de Almagro y Torres, así como algunas monedas de cecas con leyendas en signario paleohispánico del medio-alto Ebro: arsaos $^{2}$ (BDHesp Mon.37), baśkunes (BDHesp Mon.38), turiazu (BDHesp Mon.51), segeiza (BDHesp Mon.78) ${ }^{3}$ y uirouia (BDHesp Mon.71) (Alfaro 2008, 31-34).

1 Agradecemos los comentarios de Carlos Jordán, Xaverio Ballester, Javier Velaza, Blanca M. ${ }^{a}$ Prósper y de los evaluadores anónimos a una versión preliminar de este trabajo que han permitido mejorar el resultado final.

${ }^{2}$ Mantenemos la transcripción a la ibérica, tanto de arsaos como de baśkunes, con $s$ y no con $\mathbf{z}$ (Jordán 2019, 329 y 344) al no tratarse de cecas celtibéricas, sino probablemente vasconas (Jordán 2019, 327).

3 Seguimos la convención de Villar $(1993 ; 1995)$ en la representación de las sibilantes, $\mathbf{s} / \mathbf{z}$, que también se representan como s/đ según la notación de Untermann $(1997,382)$ y que corresponden a los signos transcritos ś/s en textos ibéricos. En las inscripciones redundantes se representa la vocal adicional en superíndice: tuu. Las inscripciones que consideramos escritas en escritura dual van en negrita cursiva. Mientras que las no-duales van en negrita redonda. En este último caso, a pesar de que la convención mayoritaria es transcribir las oclusivas velares y dentales de forma arbitraria como sordas. sekeiza, aun aceptando que el valor puede ser tanto el de sorda como el de sonora, optamos por se- 


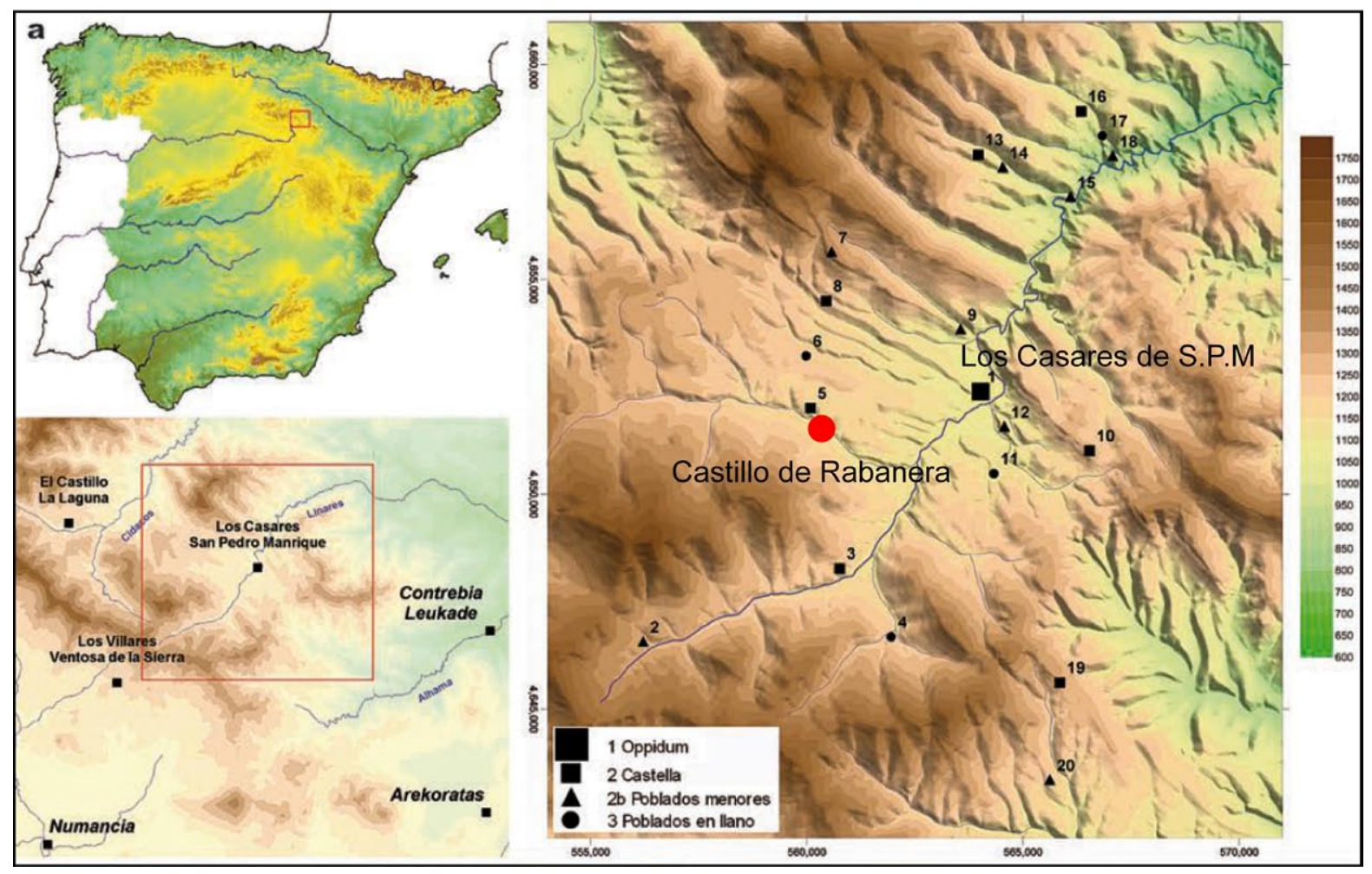

FIG. 1. Lugar de procedencia de la inscripción: Castillo de Rabanera (5).

En resumen, un yacimiento arqueológico de amplia secuencia cronológica que tiene su origen en época celtibérica como una unidad básica de poblamiento que formaba parte del territorium del oppidum de Los Casares de San Pedro Manrique controlando uno de sus valles, el del río Ventosa. Tiene la particularidad de que es uno de los pocos poblados encastillados que sobreviven a su época, la inmensa mayoría se abandonan en paralelo a los conflictos de conquista.

\section{EL SOPORTE}

Se trata de un fragmento de placa de bronce de $5,1 \mathrm{~cm}$ de largo máximo conservado y $7 \mathrm{~cm}$ de alto, actualmente de forma ligeramente trapezoidal, pero que originalmente sería rectangular. Tanto el lateral izquierdo, como el inferior y el superior son originales, mientras que el derecho

guir el criterio de usar en cada caso la sorda o la sonora de acuerdo con los paralelos disponibles, ya sea a partir de las fuentes antiguas griegas y latinas o en inscripciones duales, aun aceptando, como en el otro sistema de transcripción, que el valor puede ser tanto el de sorda como el de sonora (Ferrer i Jané 2005, notas 1 y 4). En el caso de segeiza los argumentos son favorables a que se trate de una velar sonora: $\Sigma \varepsilon \gamma \eta ́ \delta \alpha$ (Estrabón, Geog. 3.14.13) y Segidenses (Floro 1.34.3). Además, aceptamos la corrección del valor del signo leído tradicionalmente ka como variante de ke (Rodríguez-Ramos 2001-2002). 


\section{EDUARDO ALFARO PEÑA, JOAN FERRER I JANÉ, † JOAQUÍN GÓMEZ-PANTOJA FDEZ.-SALGUERO}

presenta una clara fractura. La fractura es muy irregular, por lo que no se trata de un corte realizado con alguna herramienta de corte limpio, sino empleando la fuerza. También están perdidos los ángulos superior e inferior del lateral izquierdo, en los que se aprecian los restos de sendos agujeros de $5 \mathrm{~mm}$ de diámetro que serían el mecanismo de sujeción de la placa, junto con, probablemente, otros dos que estarían situados simétricamente en el lateral derecho perdido. Los dos fragmentos perdidos en los ángulos en principio atestiguan que la placa estaba bien sujeta al soporte primario y que la única forma de liberarla fue romperla por los agujeros de sujeción. Siguiendo los bordes de la lámina, se identifican los restos de una línea de marco aproximadamente a 2,5 mm del borde, característica destacable, puesto que no la presenta ninguna de las láminas ni tabulas de bronce celtibéricas (Fig. 3). No presenta líneas de pautado, en este caso dentro de lo normal, puesto que solo una de las láminas de bronce celtibéricas, el bronce de Cortono (K.0.7) ${ }^{4}$, las presenta.

Respecto del tamaño original de la placa, solo podemos conjeturar que al tratarse de un texto escrito solo por una de sus caras y que en la última línea se aprecia el inicio de un segmento, que en media tendría plausiblemente unos cinco o seis signos, podríamos esperar una lámina aproximadamente como mínimo del doble del largo actual, es decir de unos $10,2 \mathrm{~cm}$, circunstancia que proporcionaría una ratio de 1,5 entre el lateral más largo y el más corto, que es la relación más habitual entre los documentos similares que se conservan enteros, como el bronce de Cortono (K.0.7), el bronce Res (K.0.14) y el bronce de Torrijo (BDHesp TE.03.01).

Las láminas y placas de bronce con escritura celtibérica no son muy numerosas (Jordán 2018a, 198): solo siete. Normalmente se diferencia entre láminas y placas ${ }^{5}$; las segundas serían de mayor tamańo y grosor que las primeras. Así, las tres de Botorrita se clasificarían como placas, mientras que el bronce de Cortono, el bronce Res y el de Torrijo serían láminas. Aunque a veces con discrepancia de criterios, puesto que el bronce de Luzaga sería una placa para Jordán, pero una lámina para Simón. En nuestro caso este fragmento correspondería mejor con la definición de lámina, puesto que la altura sería la segunda menor del grupo, solo por encima de la del bronce Res, y la superficie estimada sería solo superior a este último y bastante inferior tanto a la del bronce de Cortono como a la del bronce de Torrijo.

También podría pertenecer a este grupo la lámina de procedencia desconocida denominada bronce -kue ${ }^{6}$, pero tal como se argumenta más adelante, el texto sea probablemente una falsificación sobre un soporte antiguo. Además, también se excluye la lámina de pequeño tamaño de $\mathrm{Nu}$ mancia $(5,2 \times 1,5)$ y los tres pequeños fragmentos de láminas, una de Valdeherrera (K.22.1) y dos de La Custodia (Simón 2013a, 323-324). Tampoco consideramos dentro de este grupo el broche de cinturón de bronce reutilizado como soporte de escritura (K.1.2).

\footnotetext{
4 Las referencias a las inscripciones, si no se indica lo contrario, corresponden a los diferentes volúmenes de los MLH de Untermann (1980; 1990a, para las ibéricas y 1997 para las celtibéricas). El acrónimo BDHesp corresponde a la Base de Datos Hesperia y se usa para las inscripciones más recientes, no catalogadas en los MLH: http://hesperia.ucm.es/
}

\footnotetext{
5 Simón 2013, 321-324; Jordán 2018a, 201; 2019, 649-650.

6 BDHesp SP.02.16; De Hoz 1999, 458; Jordán 2019, 667.
} 


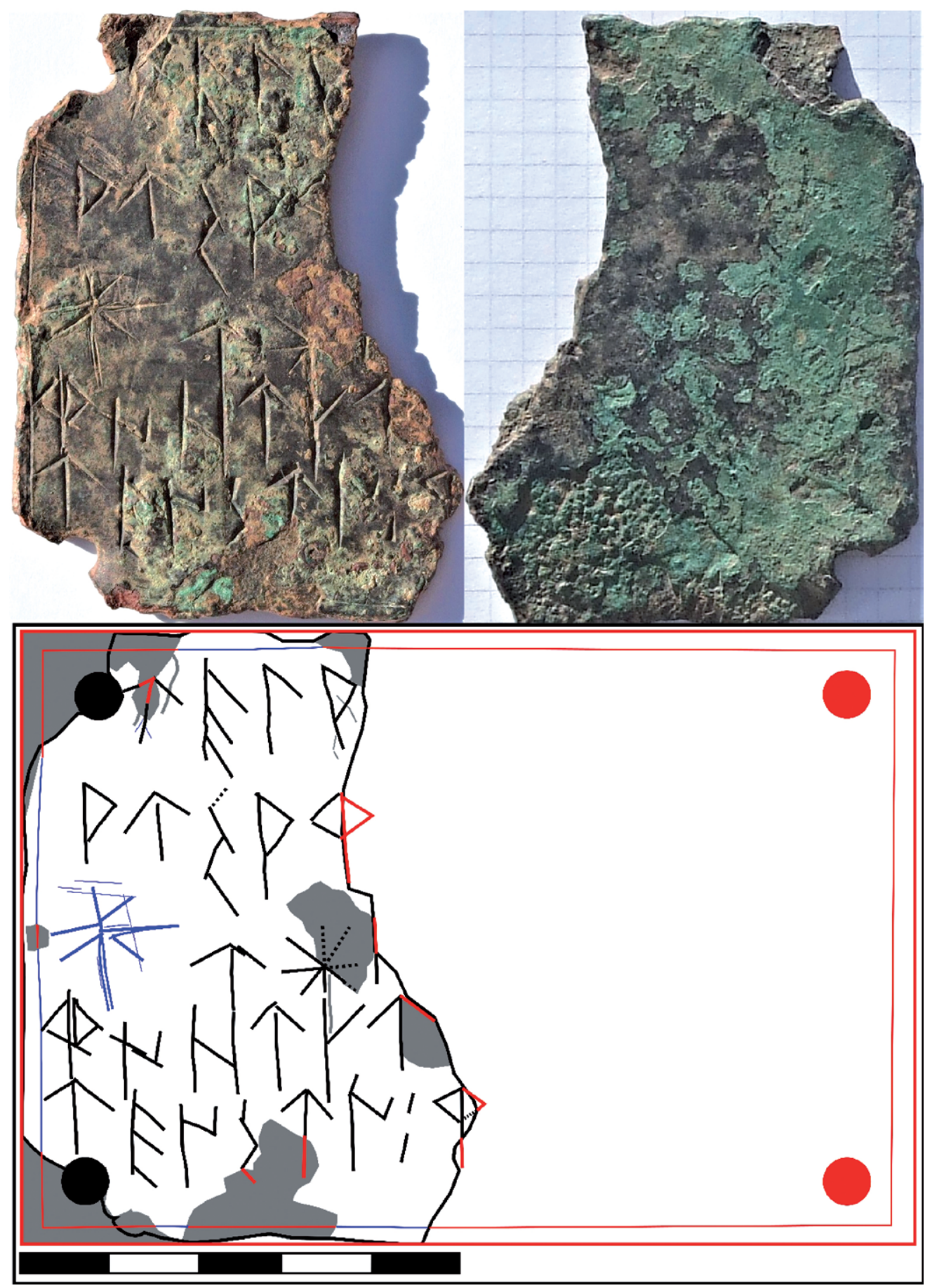

FIG. 2. Fotografías de anverso y reverso (arriba). Dibujo de la inscripción y restitución ${ }^{7}$.

7 En gris zonas erosionadas de la superficie que generalmente coinciden con los trazos perdidos, aunque a veces es posible percibir restos del trazo original, que aparecen en trazo discontinuo. 


\section{EDUARDO ALFARO PEÑA, JOAN FERRER I JANÉ, † JOAQUÍN GÓMEZ-PANTOJA FDEZ.-SALGUERO}

Los agujeros de sujeción también aparecen en otras láminas y placas de bronce. En el caso del bronce de Cortono, uno a media altura a la izquierda. En el bronce de Luzaga (K.6.1), siete orificios distribuidos irregularmente, dos de los cuales están en el interior del texto, quizás como resultado de la reutilización de la placa (Simón 2007, 225). El bronce Res no presenta orificios de sujeción, aunque en una de sus caras hay dos claros círculos incisos junto al borde, uno centrado y otro en la mitad derecha, y restos menos visibles de un tercero no dibujado en la mitad izquierda, que quizá correspondan a marcas para futuras perforaciones que no llegaron a realizarse (Burillo 19891990, 314). En el caso del tercer bronce de Botorrita (K.1.3), los orificios aparecen en dos grupos de tres, situados en la zona central superior. En cambio, ni el bronce de Torrijo ni el primer bronce de Botorrita (K.1.1) presentan restos de orificios de sujeción. No obstante, queda claro en este último caso, por el espacio exento de la cara B, que debieron existir mecanismos alternativos a los orificios de sujeción (Beltrán 1996, 59; Jordán 2018a, 204).

\begin{tabular}{|l|c|c|l|c|c|c|c|c|c|c|}
\hline \multicolumn{1}{|c|}{ Ref. } & $\begin{array}{c}\text { Año } \\
\text { Pub. }\end{array}$ & C? & YACImiento & N.o Sig. & LARGo & Alto & Sur. & R & E & $\begin{array}{c}\text { Altura } \\
\text { Signos }\end{array}$ \\
\hline K.0.14 & 1990 & S & Desc. & 134 & 7,6 & 5,1 & 39 & 1,5 & Oc & $0,5-1,3$ \\
\hline Ventosa & 2021 & N & Ventosa & 26 & $5,1(10,2)$ & 7,0 & 72 & $(1,5)$ & Oc & $0,8-1,4$ \\
\hline $\begin{array}{l}\text { BDHesp } \\
\text { TE.03.01 }\end{array}$ & 1996 & S & Torrijo & 128 & 8,8 & 13,0 & 114 & 1,5 & Or & $0,4-0,9$ \\
\hline K.0.7 & 1985 & S & Desc. & 62 & 13,6 & 8,9 & 121 & 1,5 & Or & $0,9-1,5$ \\
\hline $\begin{array}{l}\text { BDHesp } \\
\text { Z.09.24 }\end{array}$ & 1994 & N & Botorrita & 220 & 15,9 & 13,7 & 218 & & Or & 0,9 \\
\hline K.6.1 & 1882 & S & Luzaga & 122 & 16,0 & 15,0 & 240 & 1,2 & Oc & $0,6-1,2$ \\
\hline K.1.1 & 1972 & S & Botorrita & 907 & 40,5 & 10,5 & 425 & 3,9 & Or & $0,4-0.6$ \\
\hline K.1.3 & 1993 & S & Botorrita & $>3000$ & 73,2 & 51,8 & 3792 & 1,4 & Or & $0,5-1,0$ \\
\hline
\end{tabular}

TABLA 1. Cuadro comparativo de las principales dimensiones y características de las placas y láminas celtibéricas.

El criterio para diferenciar las placas de las láminas de bronce básicamente se fundamenta en la superficie del soporte y en que las de mayor superficie también suelen ser de mayor grosor. No obstante, estas diferencias en las dimensiones de los soportes no conllevan que en los soportes de mayor superficie las dimensiones de los signos sean mayores. De hecho, ocurre ligeramente lo contrario, los soportes de mayor superficie, los dos bronces de Botorrita, tienen los signos de menor altura, de $0,5 \mathrm{~cm}$ el primero y entre 0,5 y $1 \mathrm{~cm}$ el tercero, por lo que su impacto visual, estrictamente como mensaje escrito, sería similar al de las láminas. Sería el caso del bronce de Ventosa, con signos de entre 0,8 y 1,4 cm, y del bronce de Luzaga con signos entre 0,6 y 1,2 cm.

La funcionalidad precisa de estas láminas no es clara; aun así, hay un cierto acuerdo en considerar que se trata de documentos oficiales públicos ${ }^{9}$ tanto por el uso del bronce, como porque mu-

8 C? = Completo? Sup. = Superficie $=$ Largo $\times$ Alto. $\mathrm{R}=$ ratio entre el lateral más largo y el más corto. $\mathrm{E}=$ Escritura: Oriental / Occidental. Dimensiones en centímetros.
9 De Hoz 1995, 14; 1999, 459; Beltrán \& Jordán 2008, 292; Simón 2015, 28. 
chos presentan los orificios de sujeción característicos de estos documentos, y para los que no, se suponen otros mecanismos de sujeción. También las menciones de ciudades en algunos de ellos apoyan esta interpretación: gortono y gortonei en K.0.7, habitualmente relacionados con los Cortonenses de Plinio (Nat. hist. III, 3.24), también documentado en la tésera K.0.13, kortonikum, pero con argumentación en contra de Jordán $(2019,675)$. Y aregoratikubos y lutiagei en K.6.1, esta última referida probablemente a la propia Luzaga.

Para el bronce de Luzaga sí que existe un cierto consenso en que se trataría de un documento de hospitalidad destinado a ser expuesto y equivalente de las tábulas de hospitalidad latinas ${ }^{10}$. En cambio, para el bronce de Cortono se han planteado diversas hipótesis: una tésera de hospitalidad ${ }^{11}$, un documento privado (Villar 1991, 60), un pasaporte para inspeccionar minas de plata (Meid 1996, 49) y un documento oficial referente a la gestión de las minas de plata ${ }^{12}$ o a la gestión de las minas de sal (Fernández-Nieto 2001, 384).

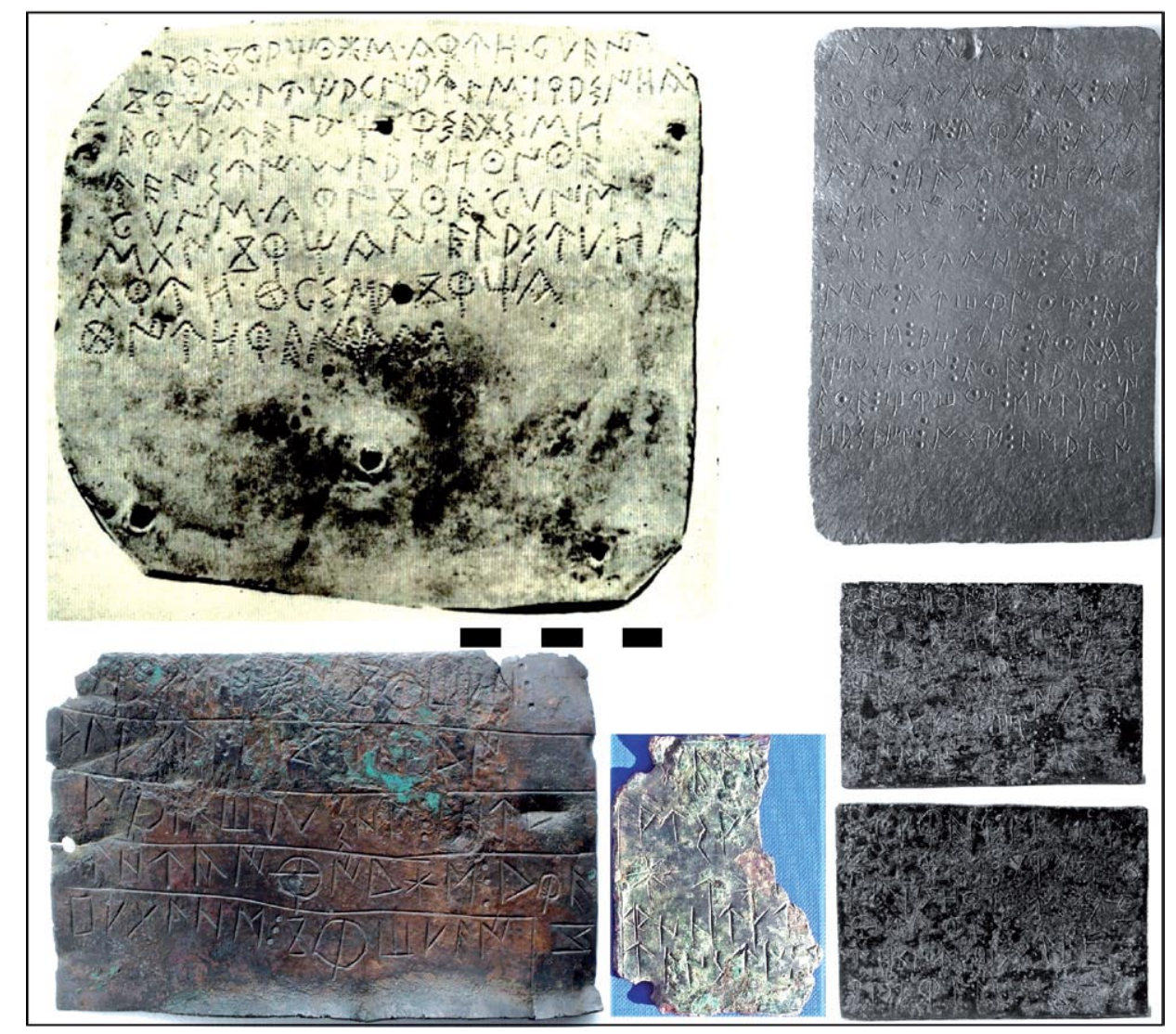

Fig. 3. Paralelos más cercanos para el bronce de Ventosa: Bronce de Luzaga, bronce de Torrijo, bronce de Cortono, bronce de Ventosa, bronce Res.

10 Tovar 1948, 75; De Hoz 1986, 76; Untermann 1990b; 1997, 375; Beltrán 1995, 178; Simón 2007, 221.
11 Fatás 1985, 425; Untermann 1990b, 367; Beltrán 1995, 178.

12 Burillo 1998, 282; De Bernardo 2004, 139; Prósper 2005, 291. 


\section{LA INSCRIPCIÓN}

El análisis epigráfico (JFJ) no se ha realizado a partir de inspección directa, sino a partir de un juego de fotografías, aunque realizadas con iluminación variable y con calidad suficiente como para tener una certeza razonable de que las lecturas propuestas son correctas.

La inscripción consta de cinco líneas con restos visibles de 26 signos de entre 0,8 y 1,4 cm: 4 en la 1. ${ }^{\mathrm{a}}$ línea, 5 en la $2 .^{\mathrm{a}}$, 4 en la $3 .^{\mathrm{a}}$, 6 en la $4 .^{\mathrm{a}}$ y 7 en la $5 .^{\mathrm{a}}$. Está realizada mediante la técnica de la incisión, como la mayoría de los paralelos sobre láminas de bronce, excepto el bronce de Luzaga y el tercer bronce de Botorrita, que usan el puntillado. Aunque probablemente la inscripción completa al menos duplicaría la parte conservada, con unos 34 signos perdidos. Los interlineados son muy irregulares y en los últimos renglones casi desaparecen, uniéndose algunos trazos de líneas contiguas y en otros casos intercalándose trazos. Los propios signos presentan líneas base muy dispares. La primera línea empieza a un $\mathrm{cm}$ del borde obligado por la presencia previa del orificio de sujeción, mientras que la segunda empieza a $0,75 \mathrm{~cm}$; el resto empieza a $0,25 \mathrm{~cm}$, usando ya como referencia la línea de marco. La primera línea empieza a $0,25 \mathrm{~cm}$ del borde superior, mientras que la inferior se queda a $0,7 \mathrm{~cm}$ del borde inferior. Las dos primeras reflejan una cierta relajación, desperdiciando espacio, mientras que las tres últimas intentan aprovecharlo al máximo. Todas estas irregularidades indican una escasa o nula planificación del texto.

La presencia del signo ḿ (n occidental) identifica claramente la inscripción como perteneciente a la escritura occidental, aunque no se detecta la presencia de la segunda nasal. No es posible precisar si se trata de la variante dual, puesto que no aparece en el texto ningún silabograma dental ni velar; el único silabograma presente es bo. Por la misma razón, tampoco se puede descartar que fuera una inscripción redundante, puesto que el signo que sigue a bo está fragmentado y los trazos conservados serían compatibles con una $\mathbf{o}$.

Sobre el origen de las dos modalidades de escritura celtibérica, oriental y occidental, la hipótesis mayoritaria considera un doble origen para justificar los dos modelos, con soluciones distintas para el uso de las nasales (cf. Ferrer i Jané 2017, 85-87), aunque Jordán (2017a, 325) defiende una monogénesis celtibérica oriental que daría lugar posteriormente a la occidental. A nuestro parecer, la simplificación de los modelos duales celtibéricos a sus variantes no-duales probablemente seguiría el mismo camino que el paso de la escritura ibérica dual a la no-dual, por el impacto socio-económico causado por la dominación romana (Ferrer i Jané 2005, 975, nota 83), aunque Jordán $(2017,326)$ considera que sería causado por una posible segunda fase de influencia ibérica, ya en fase no-dual. Por lo que respecta a la existencia de las escrituras redundantes, que reduplican el signo vocálico a continuación de los silabogramas, a nuestro parecer, sería un hecho inherente a la estructura semisilábica de las escrituras paleohispánicas y se explicaría por la existencia de escuelas epigráficas aberrantes que no habrían interiorizado el uso óptimo de la escritura semisilábica ${ }^{13}$, no obstante, para Jordán (2017a, 326), su existencia se debería a la influencia latina.

El único separador identificado tiene forma de dos pequeños trazos ligeramente diagonales a media altura, presente únicamente en la última línea, que es la más larga de las conservadas, con restos de siete signos. La ausencia de otras interpunciones y el léxico identificado apuntan a que el cambio de línea indica también el inicio de palabra, tal como sucede frecuentemente en las inscripciones celtibéricas, aunque no podemos asegurar que fuera así en todos los saltos.

13 Ferrer i Jané \& Moncunill 2020, 91; Ferrer i Jané e.p. A; e.p B. 
En principio no parece que el escriba tuviera mucha habilidad, puesto que los signos no están muy bien trazados. Además, la disposición de las líneas no es regular, especialmente las dos últimas, pues no tiene ningún sentido que estén tan juntas teniendo en cuenta el espacio sobrante final. No obstante, la solución a esta extraña disposición se explica por los restos de dos agujeros simétricamente colocados en el margen izquierdo, que estarían bloqueando las esquinas y obligarían al texto a esquivarlas. Tal como ocurre con otras placas y láminas similares, la presencia de orificios de sujeción planificados de antemano y de los clavos que los usaran conlleva declarar inhábil para la escritura la zona alrededor de cada orificio.

Así pues, la presencia del orificio en el extremo superior izquierdo ha causado que el primer signo de esta línea se sitúe a su derecha, dejando libre el ángulo superior izquierdo de la lámina. Este signo está parcialmente afectado por una erosión superficial y por la presencia de algunos trazos que parecen adventicios. Los trazos principales permitirían reconstruir un signo $\mathbf{u} \mathbf{1}^{14}$. No obstante, también sería posible reconstruir algún otro signo, si los trazos secundarios fuesen en realidad los primarios, quizás alguna variante de bi, $\mathbf{n} 1$ (m occidental) o su casi equivalente MLH III i6, tal como se indica más adelante. A pesar de ser la reconstrucción más clara, el posible signo ul quedaría ligeramente inclinado a la derecha, circunstancia extraña pues, al tratarse del primer signo, debería haberse trazado con total libertad, aunque no es precisamente un texto cuidado. Los dos siguientes son claros: e3 y $\mathbf{1 1}$. El signo final de la primera línea en algunas fotografías no se aprecia bien, pero con la luz correcta en otras se ve claramente un signo ala.

Los cuatro primeros de la segunda línea tampoco presentan problemas, a1a, u1, z2/3 y a1a. El trazo superior del posible $\mathbf{z} 3$ no es seguro, por lo que podría ser estrictamente un $\mathbf{z} 2$, aunque de no existir, quedaría sin explicación la posición desalineada por arriba con los otros signos y que llega a ocupar el espacio de los caracteres de la línea siguiente. Del último signo solo se conserva la mitad superior izquierda, pero no parece que pueda ser otra cosa que un r2a.

Al principio de la tercera línea aparece un símbolo (*) que, quizás en otras circunstancias, podría ser interpretado como un bo3 muy irregular, pero la presencia de un bo3 canónico en la misma línea permite descartar esta alternativa. De hecho, a diferencia del trazado regular del bo3, que se ha realizado con solo cuatro trazos que se extienden por los dos hemisferios, el símbolo está ejecutado con 7 trazos independientes en cada hemisferio, aunque dos están repasados, por error o poca precisión. Estos trazos tienden a converger en el punto central, pero divergen ligeramente en inclinación en cada hemisferio. Además, para conseguir la forma canónica de bo3, realizado con ocho trazos, le faltaría el trazo diagonal intermedio entre el vertical superior y el horizontal izquierdo. También se aprecian trazos de menor grosor, quizás guías descartadas para los trazos principales.

El símbolo está situado de forma perfectamente centrada en el lateral izquierdo de la lámina y su tamaño es claramente superior al de los signos que le siguen, del orden del doble. La separación respecto del signo siguiente también es del orden del doble de la normal. Aunque el trazo horizontal derecho se prolonga hasta casi la vertical de contacto con el trazo diagonal izquierdo de u, este signo se ha desplazado hacia abajo para evitar el signo $\mathbf{z}$ de la línea superior, pero probablemente se habría trazado más hacia la izquierda si estuviera asociado al símbolo. Además, los dos signos iniciales de la línea superior, especialmente a, que es mucho más corto que la otra a de la misma línea,

\footnotetext{
14 La codificación de los signos es la correspondiente a MLH IV (Untermann 1997, 443), excepto que se indique lo contrario. Para las variantes no recogidas en MLH IV, se indica la referencia correspondiente a
}

MLH III (Untermann 1990, 246). Cuando hay diversas variantes para un mismo código base se ordenan mediante letras, por ejemplo, a1a o r2a. 
parecen condicionados por su presencia, puesto que el resto de los signos se desplazan claramente hacia abajo creando un pequeño escalón entre los dos primeros y el resto. Todos estos indicios apuntan en la misma dirección: probablemente el símbolo ya estaba grabado en la lámina cuando se escribió el texto.

A continuación, aparece una u 1 clara, cuyo trazo central llega a unirse con el derecho de la o de la línea inferior, y un fragmentado, pero reconocible, bo3, cuyo trazo inferior también casi llega a colisionar con el de la ḿ 2 de la línea inferior. En el límite de la fractura se aprecia un fragmento de signo vertical, quizás a, i, o, $1, \mathbf{n}$ (con valor $/ \mathrm{m} /$ ), s, ba y bi.

La cuarta línea empieza por una r2a con un doble trazo central, que puede ser un error o una forma particular de trazado. El segundo, en principio, podría ser una $\mathbf{n} \mathbf{1}=\mathbf{m} \mathbf{1}$, pero el hecho de que el signo anterior sea una vibrante generaría una secuencia imposible rn en inicio de palabra, aunque no en posición interior. En todo caso, los paralelos léxicos del primer segmento de la quinta línea, ueizui, donde este signo aparece por duplicado, abogan por que se trate en realidad de la variante MLH III i6, no documentada aún en celtibérico, pero con cierta tradición en ibérico, como se explica en el siguiente apartado. Los siguientes signos de esta línea son claros, o3, u1, $\mathbf{m} 2=\mathbf{n} 2$ y otro $\mathbf{u} 1$ al final que está perdido en su mitad derecha.

Los signos de la última línea no presentan particularidades: u1, e3, MLH III i6, z1/3, MLH III i6, un separador de dos trazos y un r2a con la mitad derecha y la inferior perdidas. El trazo inferior de la posible $\mathbf{z} \mathbf{3}$ no es visible, pero podría haber desaparecido por la erosión de la pieza que afecta a esta zona.

En resumen, las variantes de signos usadas son: $\mathbf{u} 1, \mathrm{e} 3, \mathbf{1 1}, \mathbf{a 1} \mathbf{a}, \mathbf{a} 1 \mathbf{a}, \mathbf{u} 1, \mathbf{z} 2 / 3, \mathbf{a} 1 \mathbf{a}, \mathbf{r} 2 \mathbf{a}, \mathbf{u} 1$, bo3, ¿?, r2a, MLH III i6, o3, u1, ḿn = n2, u1, u1, e3, MLH III i6, z1/3, MLH III i6, un separador de dos trazos y $\mathbf{r} 2 \mathbf{a}$.

Además del signo MLH III i6, que se valora en el apartado siguiente, la variante más interesante paleográficamente es bo3, de cuatro trazos, que solo se usa en el bronce de Luzaga. También es interesante el e3 de tres trazos orientados hacia abajo, muy frecuente entre las inscripciones occidentales, como el propio bronce de Luzaga y el bronce de Cortono, pero también en algunas orientales como el bronce Res y el de Torrijo.

Si se confirmaran los trazos perdidos y dudosos de las dos $\mathbf{z}$, también sería destacable el uso de la variante $\mathbf{z} 3$, poco frecuente, quizás usada en el bronce de Cortono, aunque presenta un pequeño trazo que, de ser significativo, la convertiría en z5. Y quizás también en alguno de los signos z del bronce Res, no obstante, las fotografías no permiten asegurar la presencia de todos los trazos. Alternativamente, cabría admitir que se usan simultáneamente $\mathbf{z} 1$ y z2, como sucede por ejemplo en el bronce Res.

Así pues, descartando que el símbolo forme parte del texto, la lectura propuesta sería:

$$
\begin{aligned}
& \text { uela[---] / } \\
& \text { auzar[---] / } \\
& \text { ubo+[---] / } \\
& \text { riounu[---] / } \\
& \text { ueizui : r[---] }
\end{aligned}
$$

Como ya se ha indicado, los principales paralelos formales de esta inscripción son las láminas o placas de bronce, principalmente el bronce de Luzaga, el bronce Res, el bronce de Torrijo y el bronce de Cortono. Aunque por su pequeño tamaño $-7 \mathrm{~cm}$ de alto por unos aproximadamente $10,2 \mathrm{~cm}$ de largo-, si la ratio largo/alto fuera 1,5, como el que se respeta en las mencionadas lá- 
minas, los paralelos más cercanos serían el bronce Res y el bronce de Cortono, especialmente este último, por estar escrito solo por una cara y por ser de longitud similar (62 el de Cortono y unos 60 para el de Ventosa). Si las dimensiones estimadas fuesen correctas, el texto perdido no sería excesivo, probablemente una palabra en cada línea, quizás en algún caso dos si fuesen cortas, con lo que tendríamos un texto de entre 10 y 12 palabras, comparable con el del bronce de Cortono, de 12 palabras.

\section{LA FORMA ESPECIAL DEL SIGNO I}

La característica paleográfica más destacada de esta inscripción es el uso de una variante de i muy poco común en ibérico y desconocida hasta ahora en celtibérico. Las formas más habituales de este signo son MLLH III ila con los trazos delanteros en diagonal, e i1b con los trazos verticalizados. La forma que se documenta en este texto es la variante MLH III i6, que se caracteriza por presentar el trazo vertical intermedio de i1b en una posición tan elevada que puede llegar a confundirse con el trazo vertical del que aparenta ser una extensión y así puede ser escrito de un solo trazo. También en algún caso se documenta la variante formada a partir de $\mathbf{i} 1 \mathbf{b}$, en la que el trazo desplazado aún no se ha verticalizado y presenta la inclinación original. De hecho, en este texto se documentan ambas variantes: la segunda i de ueizui está formada a partir de ilb, en la que puede apreciarse que el trazo diagonal está diferenciado del vertical, mientras que las otras dos aparentemente serían una mezcla con $\mathbf{r} \mathbf{1} \mathbf{b}$, con el trazo diagonal delantero, pero en las que el trazo intermedio aparece unido al trazo vertical. Estas formas acaban siendo muy similares a la del signo $\mathbf{n}$ tradicional, nla y n 1 b, que, por efecto de un trazado defectuoso, podría presentar de manera natural la misma forma que las anteriores, si el trazo vertical acaba sobrepasando al trazo diagonal.

La variante MLH III i6 se documenta fundamentalmente en el yacimiento de Ensérune ${ }^{15} \mathrm{y}$ también en Pech Maho (B.7.3); prácticamente todas ellas son cerámicas áticas del siglo IV a.C. No obstante, esta variante se documenta también por duplicado en la inscripción rupestre de paleografia arcaica del Abrigo Burgal (BDHesp V.10.01) en la zona edetana. De hecho, la zona edetana es muy probablemente el origen de la variante ibérica en la que se inspira la escritura occidental celtibérica, por el uso de las variantes de ti y to de dos trazos, que son casi exclusivas de esta zona ${ }^{16}$. Alternativamente, como ya se ha apuntado en el apartado anterior, Jordán $(2017,325)$ defiende que la escritura oriental habría dado lugar a la occidental, dando la vuelta a la propuesta inicial de De Hoz $(1986,52)$, que proponía que la variante occidental fuera el origen de la oriental.

El uso de la variante MLH III i6 podría reforzar esta conexión; no obstante, su desarrollo también podría llegar a producirse de manera natural al trazar de forma desplazada a la izquierda el trazo normalmente en posición central. Esta línea de evolución podría estar indicada por el signo i de la estela de Trévago en escritura occidental (K.10.1) o la leyenda también occidental oilaunikos (BDHesp Mon.56), en algunas de cuyas emisiones se aprecia el mismo desplazamiento del trazo superior y que incluso en algún caso llega a unirse con el inferior. Sin embargo, en este caso, hay que tener en cuenta las dificultades de trazado de un cuño con signos de pequeńas dimensiones. Tanto la estela de Trévago como, probablemente también, las emisiones de oilaunikos se localizan en el entorno de Ventosa. No obstante, el mismo fenómeno se detecta en un esgrafiado sobre

15 B.1.3, B.1.36, B.1,37, B.1.57, B1.58, B.1.161,

16 Ferrer 2005, 975, nota 82; 2017, 86; 2019, 38. B.1.333. 


\section{EDUARDO ALFARO PEÑA, JOAN FERRER I JANÉ, † JOAQUÍN GÓMEZ-PANTOJA FDEZ.-SALGUERO}

campaniana B de Botorrita (K.1.14), en escritura oriental. En el caso del bronce de Ventosa, el tercer signo parece claramente realizado con el trazo superior diferenciado del inferior, incluso con la orientación que le correspondería; en cambio, en los otros dos signos, ambos trazos parecen realizados con el mismo golpe.

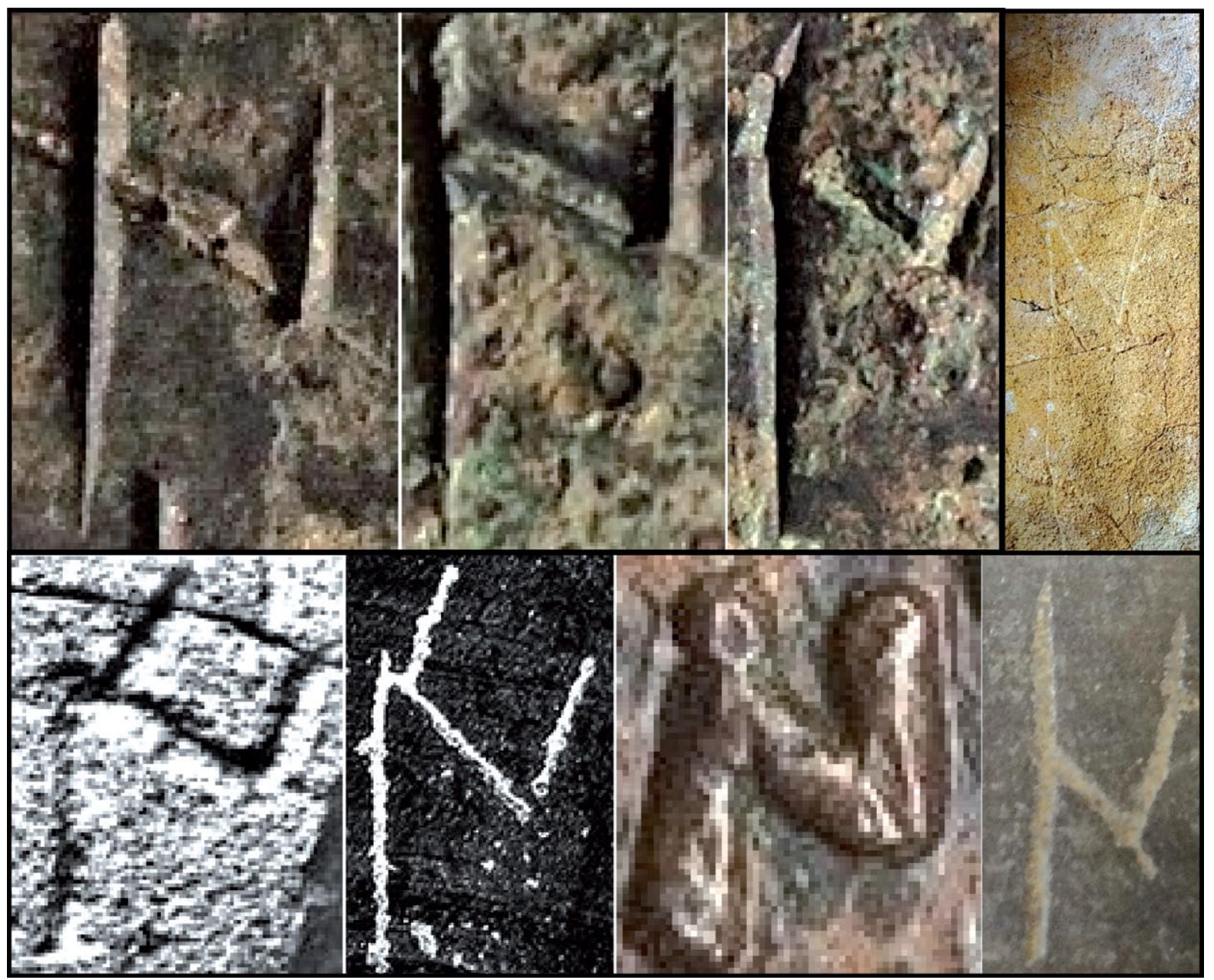

FIG. 4. Arriba: Detalle de los tres signos i6 del bronce de Ventosa y el i6 del Abrigo Burgal (BDHesp V.10.01). Abajo: El signo $i$ de K.10.1 y K.1.14 con el trazo desplazado. Un signo i6 de la leyenda oilaunikos (BDHesp Mon.56.1) y otro de Ensérune (B.1.161).

Finalmente, no debería confundirse la casuística de MLH III i6 con otros casos de duda entre $\mathbf{n}$ e i, especialmente si se trata de escritura oriental. El más conocido es el de la tésera en forma de jabalí K.0.6 que presenta el texto atulikum, aunque estrictamente lo que se transcribe es atulnkum, con un signo $\mathbf{n}$ estándar ${ }^{17}$. También se debe tener en cuenta que tanto Simón $(2013,459)$ como

17 Tovar 1983; Beltrán et al. 2009, 651; Jordán 2012; 2019, 609-617. 
Beltrán et al. $(2009,651)$ la consideran sospechosa, en parte precisamente por el problema con este signo, circunstancia que resolvería el problema, aunque Jordán $(2019,609-617)$ la trata como genuina.

\section{Cronología}

Al tratarse de una pieza procedente del hallazgo de un particular, que carece de contexto arqueológico, la determinación de su cronología debe realizarse por medios indirectos.

Respecto de la cronología general de las inscripciones celtibéricas, la identificación del uso del sistema dual en la escritura celtibérica occidental ${ }^{18}$, que entre los iberos es casi el único usado en los siglos v a.C. y III a.C., y que desaparece a principios del siglo II a.C., ya indicaba que la transmisión de la escritura de los iberos a los celtíberos se produjo muy probablemente en pleno siglo III a.C. (Ferrer i Jane 2005, 975). Esta hipótesis cronológica se ha visto recientemente confirmada con la aparición de la fálera de plata de Armuña de Tajuña ${ }^{19}$, con una inscripción explícitamente dual, puesto que se trata de un tesoro fechado a principios del siglo inI a.C. de acuerdo con las monedas presentes. No obstante, la sorpresa ha estado en que se trate de una inscripción oriental, puesto que las únicas inscripciones celtibéricas con seguridad duales eran, hasta ese momento, occidentales.

En el caso del bronce de Ventosa, tampoco el soporte en sí mismo proporciona una clara restricción cronológica, más allá de que las inscripciones celtibéricas sobre placas y láminas de bronce se sitúan, de forma general, entre finales del siglo in a.C. y mediados del siglo i a.C., por considerarse que son un producto de la influencia de la epigrafía latina una vez ya controlan el territorio celtibérico $^{20}$.

\section{LÉXICO}

La primera alternativa de lectura del primer segmento de esta inscripción es uxela[, que aparece también en el bronce de Luzaga, uela. No obstante, al estar fragmentado por la derecha, no se puede asegurar que sea estrictamente el mismo elemento. Respecto de su significado, hay un cierto consenso en considerar que tiene relación con el concepto de 'bien' o 'bueno' ${ }^{21}$. Para Meid $(1994,45)$ sería un adjetivo femenino a partir de la raíz * wel- 'bien', que estaría concertado con el elemento anterior erna, y que traduce como 'buena acogida'. Para Prósper $(2005,363)$ también sería un adjetivo femenino a partir de la raíz *welH-no-, que, en córnico, bretón y galés, está fosilizado como gwell 'mejor'.

Las alternativas secundarias de lectura biela[, iela[ (con MLH III i6) o mela[ (con $\mathbf{n}$ occidental, $=\mathbf{m}$ ), no proporcionan paralelos significativos. La única posibilidad de lograrlos sería prescindir del supuesto primer signo, suponiendo que lo que se interpretan como restos de un signo son solo fracturas, erosiones superficiales y trazos adventicios. Si se confirmara esta última hipótesis, sería posible leer ela[, que sí proporcionaría paralelos; el más destacable sería el de los antropónimos elazuno, elazuna y elazunos del tercer bronce de Botorrita, y el elazunom del bronce de Luzaga. 316.

18 Ferrer i Jané 2005, 973; Jordán 2005, 1013.

19 Velaza e.p.; Ferrer i Jané 2017, 86; Jordán 2017a,
20 De Hoz 1986, 67-68; Beltrán 1993, 254; 1995, 178; Untermann 1995, 205; Jordán 2018a, 198.

21 Gil 1977, 172; Meid 1994, 45; Wodtko 2000, 441; Prósper 2005, 363; Jordán 2019, 726. 


\section{EDUARDO ALFARO PEÑA, JOAN FERRER I JANÉ, † JOAQUÍN GÓMEZ-PANTOJA FDEZ.-SALGUERO}

El primer segmento de la segunda línea (auzar[) tendría su mejor paralelo en los dos auzares del bronce Res, que probablemente se integran en un paradigma verbal de base auz- al que podrían pertenecer también las formas siguientes, aunque para algunas de ellas algunos investigadores contemplan otras opciones (Jordán 2019, 206, 443-445): auzeti del primer bronce de Botorrita, auzanto del tercer bronce de Botorrita, auz de un pequeño elemento rectangular de bronce (K.0.8), auza[ o auz del esgrafiado antes de la cocción sobre una cerámica de Albalate del Arzobispo (K.2.1), auz en el esgrafiado de la oenochoe de Caminreal (K.5.1), y quizás también auzimei del plomo de Iniesta (BDHesp CU.00.02) (Lorrio y Velaza 2005). La última propuesta de inclusión en el paradigma es la de Jordán (2015 que añade la forma audintum del bronce de Novallas (BDHesp Z.02.01) (Beltrán et al. 2013).

Respecto del significado base del paradigma de auz-, para Untermann $(1997,640){ }^{*}$ auz $($ ez $)$ sería la 3. a persona del singular de un pretérito de la raíz * $a w d^{b}-<* h_{2} e w d^{h}-$ : 'regaló', 'dedicó', 'dio'. En la misma línea, Wodtko (2000, 49-53; 2003, 29) interpreta 'dar'. Para Schumacher (2004) estaría relacionado también con la raíz ${ }^{*} h_{2} e w d^{h}$-, responsable de la base germánica *aud- 'conceder, otorgar, donar', y que también tendría relación con el hitita peebutemi 'llevar (a)', 'conducir (a)', 'ejecutar', 'realizar', 'llevar a cabo' (Jordán 2015a, 13). Para Rubio (2013, 710) 'dar', 'poner' a partir de la raíz ${ }^{*} d e h_{3}-o^{*} d^{h} e h_{1}$-. Para Jordán $(2015,21 ; 2019,445){ }^{*}$ auz(az) sería una $3 .{ }^{a}$ persona del singular del aoristo con la raíz ${ }^{*} h_{2} e w-d^{h} e h_{1}-$ (entre otras posibilidades) 'conceder', 'otorgar' o 'donar'. Para Prósper $(2005,320)$, en el caso de auzares, tendríamos una forma verbal de la 3. a persona en pasado 'ha ofrendado' o 'ha consagrado', apoyándose en K.2.1, auza(res); mientras que auzeti y auzanto serían formas verbales de la raíz $* h_{2} e w$ - 'exigir', 'requerir', 'aceptar' (Prósper 2008), aunque algunas de estas propuestas han sido matizadas en un trabajo más reciente (Prósper 2016, 187 y 197). Para De Bernardo $(2007,65)$ su significado sería 'sacar o extraer (agua)', que procedería de la raíz ${ }^{*} h_{2}$ ews-.

Cabe destacar que Prósper $(2006,160)$ considera que las formas más simples del paradigma, estrictamente con auz, pertenecerían a un paradigma distinto que tendría relación con el avot galo, equivalente al fecit latino, y con raíz *(h)weh-, concretamente 'tejer'.

Si la restitución auzar[es se confirmase en el bronce de Ventosa, esta forma pasaría a engrosar el grupo de las formas -res, que podría estar formado por los elementos siguientes (cf. Jordán 2019, 217): los dos auzares del bronce Res, tunares y esozeres de este mismo bronce, kombalkores del primer bronce de Botorrita y quizás dures, doblemente documentado en el bronce de Torrijo y, como dures, en el de Cortono. Y quizás también por el aletu ${ }^{u}$ res de la tésera de Sasamón (K.14.1), aunque se trata de un texto occidental, probablemente dual, en el que el supuesto tures iría con sorda, a diferencia del dures del bronce de Cortono.

La hipótesis principal de interpretación es que fuesen formas verbales de perfecto con una desinencia de la $3 .^{\text {a }}$ persona del plural ${ }^{22}$. No obstante, para Untermann $(1997,409)$ serían sustantivos en función de verbos, y para Burillo (1989-1990) y Meid (1994), antropónimos a partir de la raíz * reks, que da -rix en galo, y para Pérez Vilatela (1996; 1998), apelativos que harían referencia a magistraturas. En el caso de dures (K.0.7) y alet ures (K.14.1) se ha propuesto también que se tratara de un antropónimo (Comes \& Velaza 2004) a partir de una inscripción latina de Bosnia-Herzegovina $(A E 2000,1178)$, en la que aparece como Tures Alburif(ilius).

22 Gil 1977, 171; Rubio 1996, 190; 2013, 704;

Wodtko 2000, 51; 2003, 20; Prósper 2005, 316; 2008,

77; Stifter 2006, 243; Jordán 2019, 217. 
El texto de la tercera línea, prescindiendo del símbolo, ubo+[---] permite dos interpretaciones; por una parte, la primaria es que el texto estuviese incompleto por la izquierda y fuera la continuación del final perdido de la segunda línea: [---]/ubo+[---], puesto que de esta manera permitiría la identificación de una forma familiar en -ubos , que corresponde al dativo-ablativo-locativoinstrumental plural (Jordán 2019, 186). A favor de su identificación en este bronce está el hecho de que estas formas son características de este tipo de láminas y además el signo fragmentado podría ser s, por lo que la reconstrucción ubos sería posible. Los ejemplos conocidos son aregoratikubos (K.6.1), akainakubos (K.1.1), eisubos (BDHesp TE.03.01), nouantubos (K.1.1), uetigubos (K.5.1) y loukaiteidubos (K.0.7). Aunque para los tres últimos casos, Jordán ${ }^{23}$ considera que la lectura del signo bo es realmente ta, y manifiesta dudas respecto de eisubos; creemos que tanto para los dos últimos como para eisubos, hay argumentos paleográficos que abogan por mantener la lectura bo (Ferrer i Jané e.p.). En el caso de aregoratikubos (K.6.1), parece claro que la forma se construye sobre el adjetivo con el sufijo -iko- del topónimo aregorata, por lo que se interpreta en el sentido de 'para/de los habitantes de aregorata' (Jordán 2019, 186).

No obstante, cabe indicar que mayoritariamente en este tipo de textos se evita romper una palabra entre líneas y en este mismo, en dos de las otras tres líneas con posible continuidad se identifican con seguridad elementos completos al principio de la línea: auzar[ y ueizui. La rotura de segmento entre líneas no se produce en el bronce de Luzaga, ni en el de Cortono, ni en el bronce Res, con las líneas retorcidas para evitarlo, ni en el tercer bronce de Botorrita, solo muy esporádicamente en el primero (K.1.1), donde se aprecia interés casi siempre en evitarlo, y solo se produce con cierta regularidad en el bronce de Torrijo y también en la lámina de plomo de Iniesta (BDHesp CU.00.02).

Así pues, la segunda alternativa sería considerar que el texto está completo por la izquierda: ubo+[. En este caso el único paralelo disponible es el de la base del grupo familiar ubokum al que pertenecen letondu y abulo en el primer bronce de Botorrita y sekontios en el tercero. De hecho, es el único grupo familiar que aparece citado en ambos bronces. Sin embargo, el signo fragmentado no podría ser ku, por lo que, de tratarse de esta base, tendría que formar parte de otro tipo de elemento. Teniendo en cuenta que los nombres de familia celtibéricos se forman a partir de antropónimos, como abulokum de abulo, sería plausible que ubo+[ estuviese haciendo referencia al antropónimo que habría dado origen al nombre de familia ubokum que, aun sin estar documentado, se reconstruye como *U(b)bos (Beltrán et al. 1996, 163). Esta forma encajaría con el fragmento documentado, puesto que el signo final podría ser $\mathbf{s}$, y se podría reconstruir el esperado ubos en nominativo singular, aunque

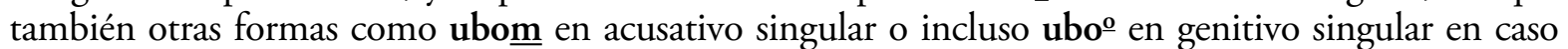
de ser escritura redundante, puesto que tanto $\mathbf{m}(=\mathbf{n})$ como o están en la relación de signos reconstruibles. La identificación del cognomen $U b i$ en una inscripción latina de Mallorca ya había hecho suponer a sus editores la relación, como genitivo latinizado, con un celtibérico ${ }^{*} \mathbf{u b o s}^{24}$.

Aunque se ha descartado la posibilidad de que el símbolo fuese una forma deficiente de bo, cabe indicar que la lectura boubo+ no produciría paralelos que la avalasen. Tampoco es productiva la alternativa de considerar que el símbolo se integra en la secuencia * ubo+ como logograma o símbolo parlante, de forma similar a como algunos investigadores interpretan el símbolo solar del bronce Res: 流的 ${ }^{25}$.

23 Jordán 2004a, 339; 2007, 104; 2017b; 2019 , $437,670,685$ y 770 .

${ }^{24}$ Hernández-Gasch et al. 2002, 57-66; AE 2002, 850; HEp 2002, 12, 15; Velaza 2014, 59-60.
25 Burillo 1989-1990, 320 y 322; Meid 1994, 47; Untermann 1997, K.0.14; Wodkto 2000; Prósper 2013-2014b, 160. 
El segmento riounu[ de la cuarta línea no tiene paralelos claros y podría ser también un segmento incompleto también por la izquierda [---]/riounu[---], aunque se aplicaría lo ya indicado para [---]/ubo+[---] respecto de la rotura de segmentos entre líneas. En todo caso, el inicio en vibrante es común en celtibérico; así, rita en una inscripción sobre recipiente de bronce de Gruissan (K.17.1), o risatioka en la primera palabra del tercer bronce de Botorrita, o ri[ en el último elemento de la inscripción sobre piedra de Trévago.

Si en la tésera de Cáceres (CIL $\left.\mathrm{I}^{2} 2825\right)$ el antropónimo fuera estrictamente Elando (Untermann 1997, 377), entonces la base del topónimo riounu podría estar relacionada con el Rian final, que Untermann interpreta como referencia al grupo familiar de Elando, o la ciudad con la que contrae el pacto, aunque también se considera posible que fuese otro antropónimo para indicar la filiación (Prósper 2005, 210).

No obstante, en el caso de que ]/riounu[ estuviera incompleto por la izquierda al continuar desde el último segmento de la línea anterior, se abrirían otras alternativas; quizás un posible $\mathbf{t u}]$ /riounu, que podría compartir el elemento turi con la leyenda de las emisiones monetales de turiazu $^{26}$ (que se identifica con la actual Tarazona), o un ti]/riounu que podría compartir la inicial tiri con tirikantam de los bronces de Botorrita (K.1.1, K.1.3 y BDHesp Z.09.24). No obstante, muchas otras reconstrucciones son posibles.

En cualquier caso, puesto que el resto de los elementos pertenecen al lenguaje formular de las láminas de bronce, en un texto de este tipo se esperaría al menos una referencia a la ciudad donde se realiza el documento y quizás también a la población de origen de un posible beneficiario o a las comunidades afectadas — si fuera el caso—, y al nombre de este o del magistrado interviniente. Así sucede en las referencias toponímicas aregoratikubos y lutiagei en el bronce de Luzaga, quizás gortono y gortonei, según la interpretación tradicional, y probablemente loukaiteidubos en el bronce de Cortono. Por lo que respecta a las referencias antroponímicas más claras, estarían bundalos en el bronce de Cortono y useizunos en el bronce de Torrijo y quizás elazunom en el bronce de Luzaga.

El mejor paralelo para riounu, que avalaría la interpretación toponímica, sería el nombre de lugar oilaunu, nominativo singular de un tema en nasal ${ }^{27}$, que aparece en denarios y también como oilaunikos, gentilicio en nominativo singular masculino, en las primeras emisiones de monedas de bronce y oilaunez, ablativo singular, en las últimas emisiones ${ }^{28}$. Y como oilaunika en una tésera de procedencia desconocida (BDHesp SP.02.27). Para la localización de esta ceca se apunta al alto valle del Ebro y, como en el bronce de Ventosa, se usa la escritura occidental. Además, a unos $35 \mathrm{~km}$ de Ventosa está Muro de Ágreda, donde se localiza la ceca de aregorata (BDHesp Mon.52), con leyenda complementaria sos, que comparte con las emisiones de oilaunu (BDHesp Mon.56), por lo que su localización en la zona reforzaría el parecido. Así pues, riounu podría ser un topónimo en nominativo singular de un tema en nasal, aunque quedaría abierta la posibilidad de identificar, entre otras, la forma riounu[bos], que podría corresponder al dativo del plural. Tampoco se puede descartar que fuese el dativo o el ablativo singular de un tema en $-o,{ }^{*}$ riounos, respectivamente riounu[i] y riounu[z] (Jordán 2019, 190).

Si el paralelo propuesto entre oilaunu y riounu fuese perfecto, habría que pensar en dos topónimos que comparten el elemento final, -unu, pero que se construyen a partir de dos bases distintas, oila y rio (o quizás (]/rio). En este sentido, hay que indicar que oilaunu se supone formado por la base *oylaun con un sufijo individualizador *-on, *oylaun-on (Jordán 2019, 302), que sería el proba-

26 BDHesp Mon.51; Jordán 2019, 305.

27 Ballester 1999b, 217; De Bernardo 2012, 56; Jor-

28 Villar 1995, 342; BDHesp Mon.56; Jordán 2019, dán 2019, 302; BDHesp Mon.56. 302. 
blemente compartido con riounu. La base oila se relaciona con *owi-la > oyla 'oveja' (Jordán 2019, $303)$, aunque para Prósper $(2005,329-330 ; 2008,59)$ se debería relacionar con ${ }^{*} o(\varphi) i$-wla-mn- 'que domina sobre'. Un posible paralelo para la base rio sería el galo *riio-, del indoeuropeo * priiio-, que significa 'libre' (Delamarre 2015), y aunque básicamente aparece en antropónimos, también aparece en algunos topónimos, como Riobe de la Tabla Peutinger, actual Châteaubleau.

Por lo que respecta a la interpretación antroponímica de riounu $/{ }^{*}$ riounos, en principio los paralelos internos celtibéricos no la avalan especialmente —únicamente quizás el ya mencionado Rian de la tésera de Cáceres (CIL I ${ }^{2} 2825$ )—, pero sí los externos. Como ya se ha indicado, Delamarre (2015) identifica el elemento galo Rio en diversos antropónimos, como Rio en Clermont (CAG 63-1) o Rionus en una inscripción de Calleva (RIB II-5, 2491) y el reconstruido *Rionus en el Rioni de Oporto $(A E$ 1997, 855). En cambio, Prósper (2005, 210-211) reconstruye un antropónimo *Rionios a partir del mismo Rioni de Oporto, aunque con otra interpretación etimológica, *Regonios 'real', que relaciona con el Rian de la tésera de Cáceres (CIL I I 2825).

Volviendo a la estela de Trévago, siendo la inscripción más cercana a la de Ventosa, a unos $25 \mathrm{~km}$, y uno de los casos con un signo i más próximo a la variante MLH III i6, se podría especular con la reconstrucción ri[oun..., en la forma conjugada adecuadamente que procediera; este último elemento de la inscripción podría indicar bien la origo del personaje —si riounu[ fuese un topónimo-, o la filiación si fuese un antropónimo.

El segmento que inicia la quinta y última línea de esta inscripción vuelve a ser un elemento muy conocido, ueizui, uno de los segmentos del bronce de Luzaga: ueizui. Este elemento pertenece a un paradigma nominal en el que se integrarían también los elementos ueizos de la tésera geométrica K.0.11, ueiziai del bronce Res y probablemente también ueidui del bronce de Cortono.

Hay un cierto consenso en considerar que el elemento construido a partir de las bases ueiz- / ueid- identificaría al magistrado o testigo garante del pacto o contrato, ya fueran téseras de hospitalidad u otro tipo de contratos ${ }^{29}$. No obstante, para Meid $(1994,44$ y 48) ueizui tendría el significado de 'visitante' o 'huésped'. Morfológicamente, ueizui / ueidui correspondería a un dativo del singular de un tema en $-o$, del que ueizos sería el nominativo ${ }^{30}$. Para Jordán $(2019,702)$ ueiziai podría ser el femenino de ueizos. Mientras que para Meid (1994, 44 y 48), ueiziai sería una derivación adjetival que, junto con mitai, traduce por 'intercambio hospitalario'.

También hay un cierto consenso en su etimología, puesto que se considera que deriva de la raíz *weid- 'ver' o 'saber'31, aunque con matices en la protoforma (Jordán 2019, 274). No obstante, Prósper $(2010-2011,72)$ no considera ueidui parte de este paradigma, pues lo ve como un dativo singular de la raíz *uexto- 'llevar' o 'transportar'.

Del probable último segmento solo se ha conservado el signo $\mathbf{r}$ inicial, circunstancia que impide su reconstrucción con un mínimo de seguridad. No obstante, sí se puede suponer que, en el caso de tratarse de un solo segmento, debería tener como máximo seis signos adicionales, para formar un elemento final de unos siete signos de acuerdo con la longitud supuesta por las dimensiones habituales de estas láminas, de 2/3 de largo respecto del alto. En el caso de que correspondiera a una referencia toponímica o antroponímica, quizás se podría tratar de un elemento con la misma base rioun- declinado adecuadamente.

29 Gorrochategui 1990, 301; Burillo 1993, 564; Villar 1995, 42; Wodtko 2003, 29; Prósper 2005, 325; Jordán 2019, 726.

30 Meid 1993, 253; Villar 1995, 53; Wodtko 2000, 437-440; De Hoz 1999, 457; Jordán 2019, 677 y 726.
31 Meid 1993, 251; Gorrochategui 1990, 301; Villar 1995, 41; De Bernardo 2001, 330; Prósper 2002, 223; Wodtko 2003, 6; De Hoz 2007, 189. 
EL SÍMBOLO SOLAR DEL BRONCE RES

Para evaluar mejor el posible significado del símbolo del bronce de Ventosa, es necesario revisar las propuestas realizadas sobre el símbolo solar del bronce Res, para ver hasta qué punto son aplicables al de Ventosa.

Los investigadores que han tratado la interpretación de este símbolo se dividen básicamente entre los que lo consideran un símbolo parlante o logograma con un determinado valor fonético complejo relacionado con el significado del símbolo ${ }^{32}$, y los que lo consideran una variante especial de uno de los signos de la escritura celtibérica ${ }^{33}$. Tanto Untermann (1997), como Wodtko (2000) y Jordán (2005-2006) mantienen abiertas ambas opciones, aunque Wodtko se decanta claramente por su uso como signo.

Así, ya el primer editor de la pieza (Burillo 1989-1990, 320 y 322) considera la posibilidad que res sea un nombre de persona compuesto con el primer elemento simbólico $=$ Soli + res = rix, que se documenta en la Galia narbonense como Solirix (CIL XII, 3005 y 3095). Para Burillo (1989-1990, 315 y 325) el texto res habría sido el primer texto escrito de la cara B e identificaría a todo el documento siguiendo los paralelos ibéricos en láminas de plomo de textos independientes perpendiculares que indican al destinatario de la carta. No obstante, los paralelos ibéricos no son aplicables en este caso, puesto que la posición vertical de estos textos corresponde a la parte visible de la lámina una vez plegada, cosa que evidentemente no sucede con este bronce. Además, tal como ya indicaba De Hoz a Burillo (1989-1990, 325), la posición inclinada del separador de tres puntos en forma de bisagra del final de la primera línea parece sugerir que el texto sigue en el margen superior, tal como se suele interpretar en la actualidad ${ }^{34}$, no obstante, otros autores lo interpretan como final de la segunda línea ${ }^{35}$.

El mismo Burillo (1989-1990, 322 y 324) recoge la propuesta alternativa de Ballester ${ }^{36}$ que considera que el símbolo solar podría ser el sinónimo de auza 'aurora' o 'sol naciente' en diversas lenguas indoeuropeas, a partir de la raíz *aus-, por lo que el elemento representado por sería el mismo auzares ya documentado doblemente en este texto. Esta misma idea la propone posteriormente Meid $(1994,53)$ circunscrita a una interpretación antroponímica de todos los elementos en -res, como Burillo, considerando que res deriva de la raíz * reks, que daría en galo la frecuente terminación -rix. Mientas que auza derivaría de *aues- 'encender' y que daría el latín aurora.

También Burillo (1989-1990, 322) recoge el acuerdo de Untermann con la interpretación del símbolo solar como elemento parlante, circunstancia que expresa ya directamente Untermann (1997, K.0.14), considerando posible que el símbolo representase la palabra celtibérica para 'sol', aunque también sugiere valores posibles para su interpretación como signo. Wodtko (2000, 301) no ve posible la relación de auza- con 'sol' o similar, y considera que, en cualquier caso, el segmento res tendría que integrarse en la misma explicación que el resto de los elementos de final

32 Burillo 1989-1990, Meid 1994 y Prósper 20132014 b.

33 Pérez Vilatela 1996; 1998 y Olcoz 2013-2014.

34 Untermann 1997, K.0.14; Pérez Vilatela 1998, 151; Jordán 2005-2006, 478; 2019, 693; BDHesp SP.02.08.

35 Wodtko 2000; Prósper 2013-2014b y Olcoz 2013-2014.

\footnotetext{
36 Ballester no llegó a tratar el tema directamente en ninguna publicación y en comunicación personal con uno de nosotros (JFJ) nos comenta que ya no considera viable esta propuesta. En primer lugar, porque considera que z solo procede de una dental, sorda o sonora, no de la sibilante (Ballester 1993-1995; 2003; Almagro et al. 2017, 172), por lo que la relación de auzares con la raíz *aus- ya no tendría sentido y, además, tampoco ve claro que se trate de un símbolo parlante.
} 
-res, por lo que debería interpretarse como una forma verbal y, por lo tanto, 沾 sería en este caso una raíz verbal abreviada.

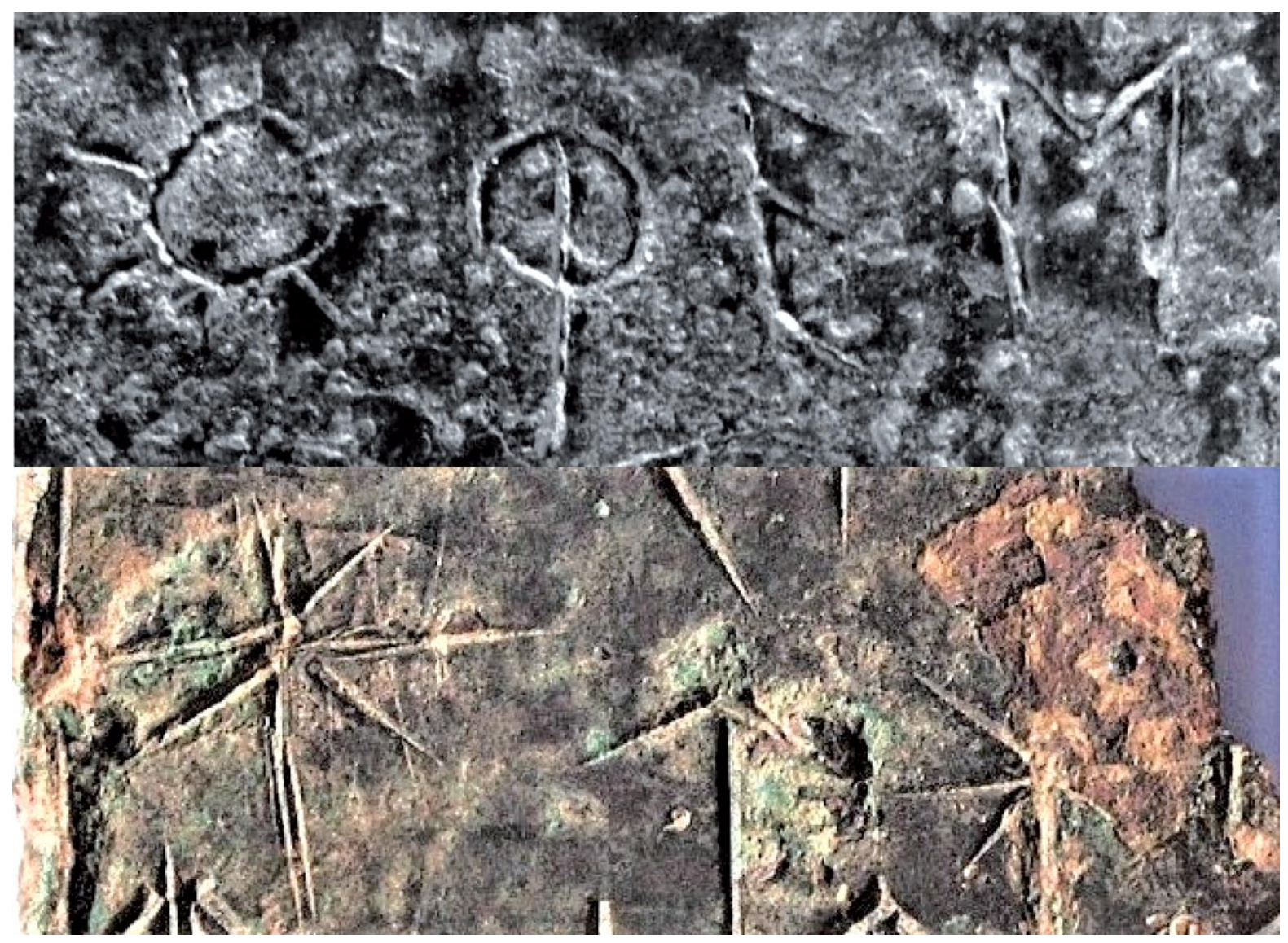

Fig. 5. Detalle de los signos que siguen al simbolo solar del bronce res: $Y$ res. $Y$ de los que siguen al simbolo solar en el bronce de Ventosa: * ubos.

Inicialmente, para Prósper $(2005,324)$ el texto siguiendo la lectura inicial de Burillo, y podría tratarse de algún tipo de firma. En una nueva propuesta, pero dentro de la interpretación simbólica, Prósper (2013-2014b, 160) considera que o res podría ser un aoristo quizás equivalente a auzares, como último elemento de la segunda línea, por lo que se le añade el signo o. Aunque no se explicita la razón de la equivalencia con auzares.

En el caso de que el símbolo solar fuese una forma particular de escribir un signo paleohispánico, ya Untermann (1997, K.0.14) considera la posibilidad que sea un alógrafo de ku o te, por su forma circular. Para Pérez Vilatela $(1998,151)$ podría ser una variante de be, por los radios que presentan las variantes arcaicas. Wodtko $(2000,300)$ se muestra más favorable a que acabe siendo una variante de un signo, más que un elemento simbólico, quizás bo, por no estar documentada en K.0.14, be, por la similitud con las variantes arcaicas MLH III be7/8/9, o incluso e, por la similitud con el signo de la escritura del sudoeste de forma similar, que se suele interpretar como va- 
riante decorada de e, pero que podría ser un hápax o variante de otro signo, como S41 (Ferrer i Jané 2016, 63) y que no tendría ningún sentido que apareciera en esta inscripción. Wodtko también considera que el signo de lectura problemática de la segunda línea podría formar parte del elemento: (o)bores, (bi)bores, (bi)eres o (bi)beres. Para Olcoz y Medrano (2013-2014, 379) sería un alógrafo de bo, aunque en la misma línea que Prósper (2013-2014b, 160, nota 8) y Wodtko (2000, 300), leen obores, considerando que 沾res pertenece a la segunda línea. También Jordán (2005-2006, 475; 2019, 706), en el supuesto de que sea una letra, considera que la única alternativa viable es que sea un signo bo del estilo Luzaga, es decir bo4, aunque lo lee como elemento final de la primera línea como bores. No obstante, desde el punto de vista de la paleografía paleohispánica todas estas propuestas, incluyendo la de interpretar el símbolo solar como un logograma, tienen muy escasa verosimilitud. Además, la mayoría de los signos propuestos ya aparecen en el texto, por lo que solo bo podría defenderse, pero su evolución desde las formas estándar a la forma solar no tendría ninguna lógica paleográfica.

Burillo también pone en relación el símbolo solar del bronce Res con los símbolos de las dos téseras de Sasamón (K.14.1 y K.14.2), pero no parece que se trate del mismo caso. En estas piezas aparecen sendos símbolos circulares (Burillo 1989-1990, 320), ambos grabados con troquel, uno con dos círculos concéntricos y un punto central (K.14.1), ocupando la posición central en la figura, y el segundo estrictamente con un círculo y un punto central (K.14.2) en el extremo inferior. Una tercera tésera anepígrafa de Sasamón tiene un símbolo similar y, en el estudio de las tres piezas, Torrija y Baquedano $(2007,335)$ les atribuyen un simbolismo astral o mágico. Un análisis más práctico es el que realizan Rodríguez y Fernández (2011), que encuentran un símbolo similar también realizado a troquel en la tésera de Ciadueńa y que atribuyen a la marca del artesano que las realizó.

En cualquier caso, como ya se ha indicado, la interpretación más probable del símbolo del bronce de Ventosa (*) es que esté grabado con anterioridad. En este caso, más que una simple decoración, o un identificativo territorial, quizás podría tratarse de algún tipo de símbolo apotropaico. Aunque en el bronce Res, aparentemente, el símbolo sí parece integrarse en el texto como un signo más, quizás se trate de un espejismo. Si fuese así, y el símbolo fuese, como en el caso del bronce de Ventosa, anterior al texto, la lectura del segmento afectado, que probablemente pertenecería a la segunda línea y no a la primera, tendría que ser ores, con el signo aislado de lectura dudosa como signo inicial y prescindiendo del símbolo. La interpretación como elemento de la primera línea con lectura res, encajaría mejor con el separador en forma de bisagra, pero dejaría el signo o aislado en la segunda línea sin explicación.

\section{CONSIDERACIONES SOBRE SU AUTENTICIDAD}

Al no proceder de una excavación arqueológica regular, la posibilidad de que se trate de una falsificación siempre debe ser considerada. No obstante, se debe matizar que esta es la situación habitual del $95 \%$ de los objetos metálicos con inscripción celtibérica, ya que excepto cinco ${ }^{37}$, el resto proceden de la actividad de búsqueda clandestina con detectores de metales o son hallazgos antiguos de particulares, muchos solo se conocen por fotografía y en casi todos consta que han circulado por el mercado de antigüedades.

37 Concretamente la tésera de Caminreal (BDHesp TE.04.11), la de Monte Bernorio (BDHesp P.05.01), la tésera de Alfaro (BDHesp LO.04.03), el primer bronce de Botorrita (K.1.1) y, con discrepancias, el tercero (K.1.3). 
En este caso, la apariencia del texto, muy poco cuidado, tanto en el grabado de los signos como en su disposición sobre la lámina, predispone de inicio a considerar la inscripción como una posible falsificación. No obstante, el análisis detallado del texto y de la paleografía, así como las particularidades del soporte, como se ha analizado en apartados anteriores, proporcionan buenas explicaciones a las aparentes irregularidades.

Así, el uso del léxico no presenta especiales sospechas, puesto que se pueden descartar las dos modalidades más típicas de falsificación. No se trata de signos colocados al azar, ya que las secuencias son perfectamente legibles, ni tampoco es una copia de ningún texto conocido. Las secuencias que se leen (uela, auzar[es] y ueizui) son coherentes con lo que sabemos de la lengua celtibérica y responden en estos tres casos a elementos bien conocidos. Tanto el símbolo como el elemento auzar[es] tienen como modelo el bronce Res, mientras que uela y ueizui tienen como modelo el bronce de Luzaga.

Puesto que el texto está roto en vertical y solo se conserva el primer elemento de cada línea, no es posible valorar cómo se interrelacionan estos elementos entre sí y con el resto del texto, circunstancia que facilitaría una hipotética falsificación del texto eligiendo elementos puntuales ya conocidos de otros textos. Así pues, de tratarse de una falsificación, sería al menos una falsificación cuidada desde el punto de vista del léxico y del uso de la escritura que intentaría representar un texto complejo plausible de forma fragmentaria. Este posible modelo de falsificación sería en principio novedoso, puesto que lo habitual es encontrar falsificaciones poco trabajadas como las de la inscripción de Bílbilis o la copia de la tésera Fröhner (Beltrán et. al. 2009, 640, CT-7), siendo la primera la concatenación de leyendas monetales que se habían transmitido deformadas por tradición manuscrita (Luján 2015), mientras que la segunda sería una muy mala copia de un texto ya conocido (Ballester 2004; Jordán 2004b, CT-7).

El soporte tampoco presenta irregularidades, siendo las roturas de las esquinas compatibles con la amortización de una placa fijada en una superficie. La probabilidad de que se trate de un soporte anepigráfico reutilizado es muy escasa, por la presencia de los restos de orificios que certifican que se trata de una placa originalmente pensada para contener un texto escrito. Tampoco la relación del texto con la superficie presenta irregularidad alguna, y se alinean bien los trazos perdidos de los signos fragmentados con las zonas erosionadas de la superficie y con la zona de la fractura.

Paleográficamente, tampoco se detecta ninguna incoherencia; siempre se usan las mismas variantes para el mismo signo, excepto la ya documentada variabilidad entre $\mathbf{z} 1$ y $\mathbf{z} 2$, si finalmente no se confirmara el uso de z3, y no se mezclan variantes cronológicamente dispares. Las variantes usadas también son las esperables en un texto celtibérico occidental, circunstancia certificada por el uso de la nasal ḿ2 $=\mathrm{n} 2(/ \mathrm{n} /)$. La localización geográfica del yacimiento de procedencia ya en la vertiente que desemboca en el Ebro se encuentra en el límite de influencia de la escritura occidental, que se solapa en esta zona con la oriental, de la que hay ejemplos hasta en la propia Numancia, en la vertiente occidental. El uso de la variante bo3 también sería coherente con la escritura occidental. Además, a favor de su autenticidad estaría el hecho que utiliza una variante del signo i (MLH III i6) que es muy rara en ibérico e inédita en celtibérico, que puede ser confundida con el signo $\mathrm{n} 1(/ \mathrm{m} /$ occidental) y que sería extremadamente raro que un falsificador usara.

Tampoco las circunstancias de su hallazgo son especialmente sospechosas, puesto que sigue en manos de un vecino del propio municipio que aparentemente la habría localizado en el yacimiento de El Castillo de Rabanera, sin que conste interés para comercializarlo en tiendas de anticuarios, por lo que el beneficio de falsificarlo decrece en gran medida. Además, al tratarse de una pieza fragmentada y con un texto breve, tampoco su valor en este mercado sería especialmente alto. 


\section{EDUARDO ALFARO PEÑA, JOAN FERRER I JANÉ, † JOAQUÍN GÓMEZ-PANTOJA FDEZ.-SALGUERO}

El problema de las falsificaciones, reales o supuestas, tiene un alto impacto en la investigación sobre el celtibérico, puesto que, del ya casi centenar de objetos metálicos con inscripción celtibérica —la mayoría bronce-, casi la cuarta parte se consideran falsos y casi otra cuarta parte, sospechosos (Jordán 2019, 503 y 507, restringido a la relación de téseras). La sospecha de falsificación de objetos con inscripciones celtibéricas se ha transformado en seguridad tras la realización de analíticas que han confirmado que algunos de los soportes estaban realizados con aleaciones con trazas de metales modernos ${ }^{38}$.

En otros casos la sospecha persiste, pues es factible complementar un objeto antiguo anepigráfico con una inscripción para aumentar su valor, como probablemente sea el caso del denominado bronce $-\mathbf{k u e}^{39}$. Aunque este bronce superó la prueba del análisis metalográfico y se considera auténtico, no presenta elementos conocidos ni interpunciones; está realizado con signos de altura homogénea y con sospechosas alineaciones verticales tanto de los signos en general como de los trazos verticales de los signos $\mathbf{i}, \mathbf{n}$ y o de la última columna. Solo la primera y la última línea aparecen desalineadas, quizás la primera para que el signo ku evite la fractura y la última para evitar usar la parte deteriorada de la esquina inferior izquierda. En particular, el signo ku es excepcionalmente grande y por su perfección parece trazado con ayuda de alguna guía o a compás. Además, el texto en general se adapta a la superficie fragmentada del soporte, de forma que no aparecen signos fragmentados en los laterales, solo alguno en la parte superior, pero con irregularidades en su forma y posición. Tal perfección formal solo parece posible con un texto inventado.

No obstante, la discrepancia en la consideración como falsas de algunas piezas es habitual, como sería el caso del bronce de Torrijo, que ha sido considerado falso por algunos especialistas (Untermann via Rubio 1999, nota 2; Wodtko 2003, 2), pero como auténtico por el resto. En este sentido, la corrección de lectura del originalmente leído kotizonei, al inesperado korzonei $\approx$ gortonei (K.0.7) ${ }^{40}$ puede considerarse prueba de su autenticidad.

También sería paradigmático el caso del bronce Res, que suscitó las dudas de algunos investigadores, especialmente de De Hoz (1999, 457), que lo excluye de su estudio y así aún aparece en Hesperia (BDHesp SP.02.08SUSPECTA), a pesar de que la mayor parte de los especialistas en celtibérico la tratan como auténtica ${ }^{41}$ y además es una de las dos inscripciones destacadas con apartado especial en el booklet AELAW sobre celtibérico (Beltrán \& Jordán 2016, 40-41).

\section{Conclusiones}

En este estudio se ha presentado la edición de un fragmento de lámina de bronce que contiene una inscripción en lengua celtibérica y escritura epicórica occidental en el que se identifican algunos de los elementos léxicos más característicos de este tipo de soportes. Se debe destacar que es la primera novedad de cierta entidad en esta tipología de inscripciones en los últimos veinte años en escritura celtibérica, circunstancia que le confiere de por sí una relevancia especial.

El bronce de Ventosa usa la escritura celtibérica occidental, aunque sería uno de los ejemplos más orientales, ya en un territorio que desagua al Ebro. No se puede determinar si se trata de una

38 Almagro 2006; Beltrán et al. 2009, 649; Jordán $2019,497$.

39 BDHesp SP.02.16; De Hoz 1999, 458; Jordán 2019, 667.
40 Ballester 2009, 35; 2013, 40; Jordán 2011, 363; 2019, 122, 675 y 688. Para Prósper 2013-2014a, 132 la equivalencia seria con ${ }^{*}$ gortionei.

41 Meid 1994; Villar 1995, 32; Pérez Vilatela 1996; 1998; Untermann 1997, 558; Wodtko 2003; Prósper 2013-2014b; Jordán 2019, 693-695. 
inscripción dual, puesto que, desafortunadamente, en el texto no aparece ningún silabograma dental ni velar, solo uno labial bo. De hecho, tampoco se puede excluir estrictamente que fuese una inscripción redundante, puesto que el signo fragmentado que sigue al silabograma bo podría ser $\mathbf{o}$.

Por lo que respecta a su cronología, el desconocimiento de su contexto arqueológico impide precisarla. No obstante, en general, las inscripciones celtibéricas se concentran principalmente en los ss. II-I a.C., aunque la fálera de Armuña se sitúa a finales del s. III a.C. y alguna de las posibles inscripciones sobre terra sigillata podría situarse ya a principios del s. I d.C. En particular, las láminas de bronce se consideran producto de la influencia romana, una vez conquistado el territorio celtibérico, por lo que deberían situarse entre finales del s. in a.C. y mediados del s. I a.C.

Desde el punto de vista paleográfico, el hecho más significativo es el uso de la variante MLH III i6, puesto que hasta ahora solo se había detectado en inscripciones ibéricas, fundamentalmente en la zona $\mathrm{B}$, pero también claramente en la zona $\mathrm{F}$ en la inscripción rupestre del abrigo Burgal (BDHesp V.10.01). Esta relación paleográfica podría incidir en la conexión entre la zona edetana y la zona celtibérica, reforzando la relación que ya se suponía por el uso de las variantes de ti y to de dos trazos. No obstante, tratándose de momento de un solo caso, no se puede descartar que este uso sea producto de una evolución paralela de esta variante a partir de las variantes clásicas, como el signo i de la inscripción K.1.14 podría sugerir.

El elemento más problemático de este nuevo texto es el símbolo de la tercera línea en el segmento * ubos, que podría estar reproduciendo la misma casuística que la ya documentada en el bronce Res en la secuencia res. No obstante, ninguna de las alternativas consideradas en la interpretación de los símbolos * y proporciona una explicación satisfactoria. Así pues, consideramos que, con los datos actuales, la alternativa preferente es que el símbolo * esté trazado con anterioridad, quizás con una función apotropaica, y que el texto lo evita.

Por lo que respecta a su autenticidad, el análisis de la paleografía y del léxico permite verificar que es compatible con el de un texto celtibérico auténtico. Los orificios de sujeción de los que quedan restos evidentes explican la extraña disposición del texto en la placa para evitarlos. También el soporte y la distribución del texto con relación a las fracturas y erosiones es convincente. Además, el uso en celtibérico de la inédita variante de i (MLH III i6), reduce considerablemente la posibilidad de falsificación, puesto que sería muy poco probable esperar que un falsificador la usara.

\begin{tabular}{|c|c|c|c|c|c|}
\hline REF. & UELA & AUZ- & 米测 & -UBOS & UEIZ- / UEID- \\
\hline BDHesp TE.03.01 & & & & eisubos & \\
\hline K.0.7 & & & & loukaiteidubos & ueidui \\
\hline K.6.1 & uela & & & aregoratikubos & ueizui \\
\hline Ventosa & uela & $\operatorname{auzar}[e s]$ & * & $\begin{array}{l}{[---] / \text { ubos? }} \\
([---] /]) \text { riounu}[\text { bos }] \text { ? }\end{array}$ & ueizui \\
\hline К.0.14 & & auzares (2) & 使 & & ueiziai \\
\hline
\end{tabular}

Tabla 2. Principales paralelos de los elementos de la inscripción.

Los principales paralelos formales de esta inscripción son las láminas o placas de bronce, principalmente el bronce de Luzaga, el bronce Res, el bronce de Torrijo y el bronce de Cortono. Aunque por su pequeño tamaño, $7 \mathrm{~cm}$ de alto por unos aproximadamente 10,2 cm de largo, si la ratio 


\section{EDUARDO ALFARO PEÑA, JOAN FERRER I JANÉ, † JOAQUÍN GÓMEZ-PANTOJA FDEZ.-SALGUERO}

largo / alto fuera de 2/3, los paralelos más cercanos serían el bronce Res y el bronce de Cortono. Especialmente este último, por estar escrito solo por una cara y tener una longitud similar (62 signos y 12 palabras el de Cortono, y unos 60 signos y supuestamente entre 10 y 12 palabras el de Ventosa, del que se habría perdido algo más de la mitad del texto).

Los paralelos de los elementos léxicos identificados, uela[, auzar[, -ubos y ueizui apuntan principalmente a las mismas inscripciones. Del bronce Res: los dos auzares, ueiziai y el símbolo. Del bronce de Luzaga: uela, ueizui y aregoratikubos. Del bronce de Cortono: ueidui y loukaiteidubos. Y del bronce de Torrijo, solo eisubos.

Entre los elementos léxicos identificados, a pesar de las dudas de lectura del primer signo, unela sería claro, puesto que hay unanimidad en interpretarlo como adjetivo femenino con el significado de 'buena' a partir de la raíz *wel- 'bien'. No obstante, el único paralelo, en el bronce de Luzaga aparece en la tercera línea, mientras que en el bronce de Ventosa encabeza el texto.

La opinión mayoritaria considera que las formas con base auz- serían formas verbales y, en concreto, para la secuencia auzar[es], la hipótesis principal es que correspondería a la tercera persona del plural de una forma de pasado con el significado dentro del entorno de 'donaron', 'ofrecieron', 'dedicaron', 'consagraron', 'dieron', 'concedieron', 'otorgaron' o 'realizaron'.

Para las formas con final en -ubos, como sería el caso de [---]/ubos de la segunda y tercera línea, se acepta con cierta unanimidad que corresponderían al dativo-locativo-ablativo del plural, que estaría formado en algunos ejemplos muy claramente a partir de un nombre de ciudad para identificar a sus habitantes, como sería el caso en el bronce de Luzaga de aregoratikubos respecto de aregorata. En cambio, la alternativa de lectura ubos, permitiría identificar el antropónimo base sobre el que se habría formado el grupo familiar ubokum.

El único elemento de este texto que no proporciona ningún paralelo claro es riounu[, o quizás /] riounu[. Las dos hipótesis principales de interpretación son que se trate de un topónimo o de un antropónimo, identificando alguna de las comunidades, ciudades o personas intervinientes en el documento. No obstante, a pesar de lo bien conocida que es la antroponimia celtibérica, no aparecen claros paralelos internos (quizás el Rian de la tésera de Cáceres, y en la antroponimia gala los de base Rio). En el campo de la toponimia, más susceptible a la aparición de nuevos elementos, riounu recordaría a la ya conocida por emisiones monetales oilaunu (BDHesp Mon.56), con la que compartiría la secuencia final: rio + unu / oila + unu. La base rio del topónimo podría corresponder a la mencionada base gala Rio que también forma parte de algún topónimo, como Riobe (Châteaubleau). Al estar incompleta por la izquierda, aun en el contexto de un topónimo de base rioun, la forma concreta presente podría ser diversa, en particular podría ser otra forma de dativo-locativo-ablativo: riounu[bos]. La localización de oilaunu no es conocida, pero comparte la leyenda sos con las de aregorata (BDHesp Mon.52), localizada habitualmente en Muro de Ágreda (Soria) a solo unos $35 \mathrm{~km}$ de Ventosa, por lo que podría ser un topónimo cercano.

El último elemento identificado en el texto es ueizui, que también suscita un cierto consenso en su etimología, puesto que se considera que deriva de la raíz *weid- 'ver' o 'saber', y se le considera como un apelativo conjugado en dativo singular que identificaría al magistrado o testigo garante del contrato o pacto.

Finalmente, en referencia a su contenido, si las propuestas semánticas de los elementos léxicos identificados son las correctas, se esperaría un texto oficial que entre otras informaciones identificara de alguna forma a la autoridad emisora (auzar[es]) del documento, las personas (ubos y/o ${ }^{*}$ riounos), ciudades (riounu) o comunidades involucradas ([---]/ubos y/o riounu[bos]), el objeto del documento y el testigo o magistrado (ueizui) que certificó la oficialidad del acto, aunque quizás 
algunos de estos datos pudieran estar implícitos por el contexto en el que la lámina estaba expuesta, dado que el texto no aparenta ser muy largo. No obstante, se debe tener presente que la funcionalidad de las láminas de bronce celtibéricas no es especialmente clara y es muy probable que sea variada. El único punto de consenso es considerar que tienen un cierto carácter oficial, especialmente por usar como soporte el bronce, y en algunos casos, como el bronce de Ventosa, por presentar indicios de que estaban expuestos públicamente.

\section{BibLIOGRAFÍA}

$A E=$ L'année épigraphique, Paris, 1888 .

Alfaro Peña, E., 2005, Castillejos y Villares. Modelos de poblamiento antiguo en el interior del Sistema Ibérico, Soria: Soria Edita.

Alfaro Peña, E., 2008, «Reliquias celtibéricas en Tierras Altas: dos fíbulas zoomorfas y un fragmento ancoriforme», Revista de Soria 61, 27-42.

Almagro, M., 2006, «Precisiones y correcciones sobre algunas téseras de la Real Academia de la Historia», PalHisp 6, 281-293.

Almagro, M., X. Ballester \& M. Turiel, 2017, «Tésera celtibérica con 'lobo cenital' procedente de Burgos», BSAA Arqueología LXXXIII, 157-185.

BDHesp = Banco de Datos Hesperia, http://hesperia.ucm.es/

Ballester, X., 1993-1995, «Sobre el valor de 4 en celtibérico», Kalathos 13-14, 319-323.

Ballester, X., 1999, «Tres notas celtibéricas: *OILAUNICa CaR, *ARGAILICA CAR y CAAR *SALMANTICA», Veleia 16, 217-220.

Ballester, X., 2003, «Celtibérico SECoNZOS = ¿Secundus o SECoNTiOS?», Veleia 20, 351-354.

Ballester, X., 2004, «Notas a epígrafes celtibéricas de colecciones particulares», PalHisp 4, 265-282.

Ballester, X., 2009, «Filología arqueoibérica: cuestión de método», en: F. Beltrán, J. D’Encarnação, A. Guerra, C. Jordán (eds.), Acta Palaeohispanica X. Actas do X Colóquio sobre Linguas e Culturas PaleoHispânicas (Lisboa, 26-28 de Fevereiro de 2009) = PalHisp 9, 23-38.

Ballester, X., 2013, «Escolios a un topónimo prerromano implícito», en: X. Ballester, F. Beltrán, F. J. Fernández Nieto, C. Jordán, J. Siles, Acta Palaeohispanica XI. Actas del XI Coloquio internacional de Lenguas y Culturas Prerromanas de la Peninsula Ibérica (Valencia, 24-27 de octubre de 2012) [= PalHisp 13], Zaragoza, 33-47.

Beltrán, F., 1993, «La epigrafia como índice de aculturación en el valle medio del Ebro (siglo in a.e.-II d.e.)", en: Lengua y cultura en la Hispania prerromana, Actas del V Coloquio sobre lenguas y culturas prerromanas de la Peninsula Ibérica (Colonia, 25-28 de noviembre de 1989), 235-272.

Beltrán, F., 1995, «La escritura en la frontera. Inscripciones y cultura epigráfica en el valle medio del Ebro", en: Roma y el nacimiento de la cultura epigráfica en Occidente, 169-195.

Beltrán, F., 1996, «useisu aiankum tauro non erat bintis. Una nota de lectura sobre la cara B de Botorrita 1», en: F. Villar, J. D’Encarnação (eds.), La Hispania prerromana. Actas del VI Coloquio sobre Lenguas y Culturas Prerromanas de la Peninsula Ibérica (Coimbra, 13-15 de octubre de 1994), Salamanca, 51-63.

Beltrán, F., J. De Hoz \& J. Untermann, 1996, El tercer bronce de Botorrita (Contrebia Belaisca), Zaragoza.

Beltrán, F., C. Jordán \& I. Simón, 2009, «Revisión y balance del 'corpus' de téseras celtibéricas», en: F. Beltrán, J. d'Encarnação, A. Guerra, C. Jordán (eds.), Acta Palaeohispanica X. Actas do X Colóquio internacional sobre Linguas e Culturas Paleohispânicas (Lisboa, 26-28 de Fevereiro de 2009) [= PalHisp 9], Zaragoza, 625-668.

Beltrán, F., \& C. Jordán, 2008, «La epigrafía pública celtibérica», en: Las culturas del Próximo Oriente Antiguo y su expansión mediterránea, 289-320.

Beltrán, F., \& C. Jordán, 2016, Celtibérico. Lengua. Escritura. Epigrafia, AELAW Booklet 1. 


\section{EDUARDO ALFARO PEÑA, JOAN FERRER I JANÉ, † JOAQUÍN GÓMEZ-PANTOJA FDEZ.-SALGUERO}

Beltrán, F., J. J. Bienes, J. A. Hernández \& C. Jordán, 2013, «El bronce celtibérico en alfabeto latino de Novallas (Zaragoza). Avance», en: X. Ballester, F. Beltrán, F. J. Fernández Nieto, C. Jordán, J. Siles, Acta Palaeohispanica XI. Actas del XI Coloquio internacional de Lenguas y Culturas Prerromanas de la Peninsula Ibérica (Valencia, 24-27 de octubre de 2012) [= PalHisp 13], Zaragoza, 615-635.

De Bernardo, P., 2001, "Grafemica e fonologia del celtiberico», en: F. Villar y M. a P. Fernández (eds.), Religión, Lengua y Cultura Prerromanas de Hispania. Actas del VIII Coloquio sobre Lenguas y Culturas Prerromanas de la Peninsula Ibérica (Salamanca, 11-15 de mayo de 1999), Salamanca, 319-334.

De Bernardo, P., 2004, «Cib. oboi 'sea eso'... alaboi 'o bien sea'...: Morfosintaxis céltica en el Bronce de Córtono (K.0.7, Ll. 1-2)», PalHisp 4, 135-151.

De Bernardo, P., 2007, "Cib. auzu 'haurio', auzeti 'haurit', auzanto 'hauriant': Water in the Botorrita bronzes and other inscriptions (K.0.8, 1.1, 1.3, 2.1, 5.1)», PalHisp 7, 55-69.

De Bernardo, P., 2012, «Reinterpreting some documents of the Celtiberian and other Palaeohispanic corpora», PalHisp 12, 51-71.

Burillo, F., 1989-1990, «Un nuevo texto celtibérico: El bronce 'Res'», Kalathos 9-10, 313-331.

Burillo, F., 1993, "Una tésera de Arekorata. Un nuevo concepto volumétrico en las téseras de hospitalidad celtibéricas", en: Homenaje a M Tarradell, Barcelona, 559-567.

Burillo, F., 1998, Los celtiberos. Etnias y estados, Barcelona.

Comes, R., \& J. VelazA, 2004, «Nota onomástica hispano-dalmática: sobre AE 2000, 1178 y MLH IV, K.0.7,4 (y K.1.3,II-37)», Orbis Antiquus. Studia in honorem Ioannis Pisonis, Cluj-Napoca, 46-47).

Delamarre, X., 2015, «Affranchis, chevaux sauvages, libérateurs et mercenaires : le mot gaulois pour 'libre'», Études Celtiques 41, 129-144.

Fatas, G., 1985, «Una tésera cortonense», en: J. L. Melena (ed.), Symbolae L. Mitxelena septuagenario oblatae, Vitoria, 425-431.

Fernández Nieto, F. J., 2001, «Revisión histórica del Bronce de Cortono», en: F. Villar, M. a P. Fernández (eds.), Religión, Lengua y Cultura Prerromanas de Hispania. Actas del VIII Coloquio sobre Lenguas y Culturas Prerromanas de la Peninsula Ibérica (Salamanca, 11-15 de mayo de 1999), Salamanca, 373-387.

Ferrer i Jané, J., 2005, «Novetats sobre el Sistema Dual de Diferenciació Gràfica de les Oclusives Sordes i Sonores», en: F. Beltrán, C. Jordán, J. Velaza (eds.), Acta Palaeohispanica IX. Actas del IX Coloquio sobre Lenguas y Culturas Paleohispánicas (Barcelona, 20-24 de octubre de 2004) [= PalHisp 5], Zaragoza, 957982.

Ferrer i Jané, J., 2016, «Una aproximació quantitativa a l'anàlisi de l'escriptura del sud-oest», PalHisp 2016, 39-79.

FERrer I JANÉ, J., 2017, "Algunas reflexiones sobre la genealogía de las escrituras paleohispánicas», en: F. Beltrán, B. Díaz, M. J. Estarán, C. Jordán, A. Klöckner, T. Schattner (eds.), Acta Palaeohispanica XII. Bild und Schrift. Medienkombinationen in den eisenzeitlichen Kulturen Hispaniens. Kolloquium zu ehren von Jürgen Untermann (Giessen, 9-12 de abril de 2016), [= PalHisp 17], Zaragoza, 55-94.

Ferrer i Jané, J., 2019, "A la recerca del trial: les variants supercomplexes a les escriptures paleohispàniques», PalHisp 19, 27-53.

Ferrer i Jané, J., e.p. A, «Las escrituras epicóricas de la Península Ibérica», PalHisp 20.

Ferrer i Jané, J., e.p. B, «El sistema dual de la escritura celtibérica desde la perspectiva ibérica», PalHisp 21.

Ferrer i Jané, J., \& N. Moncunill, 2019, «Palaeohispanic writing systems: classification, origin and development», en: J. Velaza, A. G. Sinner (eds.), Palaeohispanic Languages and Epigraphies, Oxford, 78-108.

GiL, J., 1977, «Notas a los bronces de Botorrita y de Luzaga», Habis 8, 161-172.

Gorrochategui, J., 1990, «Consideraciones sobre la fórmula onomástica y la expresión del origen en algunos textos celtibéricos menores», en: F. Villar (ed.), Studia indogermanica et palaeohispanica in honorem A. Tovar et L. Michelena, Salamanca, 291-312.

$H E p=$ Hispania Epigraphica, Madrid.

Hernández Gasch, J., J. Sanmartí \& J. Velaza, 1999, «El cuenco esgrafiado con epígrafe latino del santuario talayótico de Sa Punta des Patró (Mallorca)», Mayurqa 25, 135-138. 
Hoz, J. DE, 1986, «La epigrafía celtibérica», Actas de la Reunión sobre Epigrafía Hispánica de época romanorepublicana, Zaragoza, 41-102.

Hoz, J. DE, 1995, «Las sociedades celtibérica y lusitana y la escritura», AEspA 68, 3-30.

Hoz, J. DE, 1999, «Los metales inscritos en el mundo griego y periférico y los documentos celtibéricos en bronce», en: F. Villar, F. Beltrán (eds.), Pueblos, Lenguas y Escrituras en la Hispania Prerromana. Actas del VII Coloquio sobre Lenguas y Culturas Paleohispánicas (Zaragoza, 12 a 15 de marzo de 1997), Salamanca, 433-470.

Hoz, J. DE, 2007, «The institutional vocabulary of the Continental Celts», en: P.-Y. Lambert, G.-J. Pinault (eds.), Gaulois et celtique continental, Genève, 189-214.

Jordán, C., 2004a, Celtibérico, Zaragoza.

Jordán, C., 2004b, "Chronica Epigraphica Celtiberica III», PalHisp 4, 285-323.

Jordán, C., 2005, «¿Sistema dual de escritura en celtibérico?», en: F. Beltrán, C. Jordán, J. Velaza (eds.), Acta Palaeohispanica IX. Actas del IX Coloquio sobre Lenguas y Culturas Paleohispánicas (Barcelona, 20-24 de octubre de 2004) [= PalHisp 5], Zaragoza, 1013-1030.

Jordán, C., 2005-2006, "Consideraciones paleo-epigráficas a propósito del Bronce Res», Kalathos 24-25, 475-486.

Jordán, C., 2007, «Estudios sobre el Sistema Dual de Escritura en Epigrafia no Monetal Celtibérica», PalHisp 7, 101-142.

Jordán, C., 2011, «Relecturas de la Estela de Langa [K.12.1], del Bronce de Torrijo y el Bronce de Cortono [K.0.7]», en: M. ${ }^{a}$ J. García Blanco, T. Amado, M. ${ }^{a}$ J. Martín, A. Pereiro, M. E. Vázquez

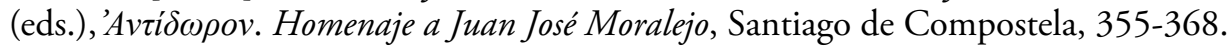

JorDÁn, C., 2012, «La interpretación morfológica de la tésera celtibérica K.0.6 y los derivados de temas en nasal en celtibérico", Emerita LXXX 1, 31-43.

Jordán, C., 2015, «Avdintvm, una nueva forma verbal en celtibérico y sus posibles relaciones paradigmáticas (auzeti, auzanto, auz, auzimei, auzares...)», Estudios griegos e indoeuropeos 25, 11-23.

Jordán, C., 2017a, «Sistema dual y redundante en celtibérico», en: F. Beltrán, B. Díaz, M.a J. Estarán, C. Jordán, A. Klöckner, T. Schattner (ed.), Acta Palaeohispanica XII. Bild und Schrift. Medienkombinationen in den eisenzeitlichen Kulturen Hispaniens. Kolloquium zu ehren von Jürgen Untermann (Giessen, 9-12 de abril de 2016), [= PalHisp 17], Zaragoza, 315-327.

JoRDÁN, C., 2017b, «Una nueva lectura e interpretación de la inscripción celtibérica sobre oinochoe [K.5.1]», en: Ratna, Homenaje a la profesora J. Mendoza, Madrid, 103-107.

Jordán, C., 2018a, "Los bronces celtibéricos» en: F. Beltrán, B. Díaz (eds.), El nacimiento de las culturas epigráficas en el occidente mediterráneo, 195-230.

Jordán, C., 2019, Lengua y Epigrafía celtibéricas, Monografías de Filología griega 29, Zaragoza.

Lorrio, A., \& J. VelazA, 2005, «La primera inscripción celtibérica sobre plomo», en: F. Beltrán, C. Jordán, J. Velaza (eds.), Acta Palaeohispanica IX. Actas del IX Coloquio sobre Lenguas y Culturas Paleohispánicas (Barcelona, 20-24 de octubre de 2004) [= PalHisp 5], Zaragoza, 1031-1048.

LujÁN, E. R., 2015, «La inscripción "celtibérica” del cerro de Bámbola (Calatayud)», en: G. Oudaer, G. Hily, H. Le Bihan (eds.), Mélanges en l'honneur de Pierre-Yves Lambert, Rennes, 299-311.

MeID, W., 1993, "Keltiberisch ueisoś und Verwandtes», en: F. Heidermarms, H. Rix, E. Seebold (eds.), Sprachen und Schriften des antiken Mittelmeerraums». Festschrift für Jürgen Untermann zum 65. Geburtstag, Innsbruck, 251-254.

MeID, W., 1994, Celtiberian Inscriptions, Budapest.

MeID, W., 1996, Kleinere keltiberische Sprachdenkmäler, Innsbruck.

Olcoz, S., \& M. Medrano, 2013-2014, «Revisión paleográfica de varias inscripciones celtibéricas de los valles del Jiloca y Huerva», Kalathos 26-27, 367-398.

Pérez Vilatela, L., 1996, "Interpretación de la cara A del "bronce res"», Emerita LXIV, 31-43.

Pérez Vilatela, L., 1998, «Propuestas para la interpretación del bronce ŕeś», Caesaraugusta 74, 133-166.

Prósper, B. M. a , 2002, «La gran inscripción rupestre celtibérica de Peñalba de Villastar. Una nueva interpretación", PalHisp 2, 213-226. 


\section{EDUARDO ALFARO PEÑA, JOAN FERRER I JANÉ, † JOAQUÍN GÓMEZ-PANTOJA FDEZ.-SALGUERO}

Prósper, B. M.a , 2005, "Estudios sobre la fonética y la morfología de la lengua celtibérica», en: F. Villar, B. M. ${ }^{\text {a }}$ Prósper, Vascos, celtas e indoeuropeos. Genes y lenguas, Salamanca, 153-364.

Prósper, B. M.a , 2006, "Un paralelo léxico-sintáctico entre celtibérico y galo: la firma de alfarero gala AVOT y celtibérico 'auz'", PalHisp 6, 151-163.

Prósper, B. M. a 2008, El Bronce celtibérico de Botorrita I, Roma-Pisa: Fabrizio Serra Editore.

Prósper, B. M. a, 2010-2011, «The Hispano-Celtic Divinity ILVRBEDA, Gold Mining in Western Hispania and the Syntactic Context of Celtiberian arkatobezom 'Silver Mine'", Die Sprache 49.1, 53-83.

Prósper, B. M.a , 2013-2014a, «Time for Celtiberian dialectology: Celtiberian syllabic structure and the interpretation of the bronze tablet from Torrijo del Campo, Teruel (Spain)», Keltische Forschungen 6, 115155.

Prósper, B. M.a , 2013-2014b, "Some notes on the structure and meaning of the bronze 'Res'", Keltische Forschungen 6, 157-164.

Prósper, B. M. a, 2016, The Indo-European Names of Central Hispania. A Study in Continental Celtic and Latin Word Formation, Innsbruck: IBS.

Rodríguez, J., \& F. Fernández, 2011, «Una nueva tésera celtibérica, procedente de Ciadueña (Soria)», PalHisp 11, 265-282.

Rodríguez Ramos, J., 2001-2002, «Okelakom, Sekeida, Bolsken», Kalathos 20-21, 429-434.

Rubio, F. J., 1996, «Tensiones paradigmáticas en el verbo celtibérico: audeti, audanto, audares y otras formas emparentadas", Kalathos 15, 181-194.

Rubio, F. J., 1999, «Aproximación lingüística al bronce de Torrijo (Teruel)», Veleia 16, 137-157.

Rubio, F. J., 2013, "Hacia la identificación de paradigmas verbales en las inscripciones celtibéricas», en: X. Ballester, F. Beltrán, F. J. Fernández Nieto, C. Jordán, J. Siles, Acta Palaeohispanica XI. Actas del XI Coloquio internacional de Lenguas y Culturas Prerromanas de la Peninsula Ibérica (Valencia, 24-27 de octubre de 2012) [= PalHisp 13], Zaragoza, 699-715.

Schumacher, S., 2004, Die keltischen Primärverben, Innsbruck.

Simón, I., 2007, «Muko · kaiko, relectura de K.9.1», PalHisp 7, 219-236.

Simón, I., 2013, Los soportes de la epigrafia paleohispánica. Inscripciones sobre piedra, bronce y cerámica, Zaragoza.

Simón, I., 2015, «La Epigrafía antigua del Jiloca», Ibérica 4, 1-129.

Stifter, D., 2006, «Contributions to Celtiberian etymology II», PalHisp 6, 237-245.

Torija, A., \& I Baquedano, 2007, «Las tesserae de la Colección Cerralbo. Viejas conocidas, nuevas perspectivas", PalHisp 7, 269-336.

Tovar, A., 1948, «El bronce de Luzaga y las téseras de hospitalidad latinas y celtibéricas», Emerita XVI, 75-91.

Tovar, A., 1983, "Una nueva pequeña tésera celtibérica», Emerita LI, 1-3.

Untermann, J., 1980, Monumenta Linguarum Hispanicarum, II Die Inschriften in iberischer Schrift aus Südfrankreich, Wiesbaden, Dr. Ludwich Reichert Verlag (= MLH II).

Untermann, J., 1990a, Monumenta Linguarum Hispanicarum, III Die iberischen Inschriften aus Spanien, Wiesbaden, Dr. Ludwich Reichert Verlag (= MLH III).

Untermann, J., 1990b, "Comentarios sobre inscripciones celtibéricas 'menores'», en: F. Villar (ed.), Studia indogermanica et palaeohispanica in honorem A. Tovar el L. Michelena, Salamanca, 351-374.

UnTERmanN, J., 1995, «Epigrafía indígena y romanización en la Celtiberia», en: F. Beltrán (ed.), Roma y el nacimiento de la cultura epigráfica en Occidente, Zaragoza: Institución «Fernando el Católico», 197-208.

Untermann, J., 1997, Monumenta Linguarum Hispanicarum IV Die tartessischen, keltiberischen und lusitanischen Inschriften, Wiesbaden, Dr. Ludwich Reichert Verlag (= MLH IV).

VeLAZA, J., 2014, «Antroponimia y lenguas prerromanas en las islas Baleares», Emerita LXXXII 1, 51-67.

VelazA, J., e.p., «Inscripción celtibérica sobre falera procedente de Armuña de Tajuña (GU)».

Villar, F., 1991, «Le locatif celtibérique et le caractère tardif de la langue celtique dans l'inscription de Penalba de Villastar», Zeitschrift für celtische Philologie 44, 56-66. 
Villar, F., 1993, «Las silbantes en celtibérico», en: J. Untermann y F. Villar Lengua y cultura en la Hispania Prerromana, en: J. Untermann y F. Villar (eds.), Lengua y cultura en la Hispania prerromana. Actas del $\checkmark$ Coloquio sobre Lenguas y Culturas Prerromanas de la Peninsula Ibérica (Colonia, 25-28 de noviembre de 1989), 773-818.

VIllar, F., 1995, Estudios de celtibérico y de toponimia prerromana, Salamanca.

Wodtкo, D. S., 2000, Monumenta Linguarum Hispanicarum V.1. Wörterbuch der keltiberischen Inschriften, Wiesbaden.

Wodтко, D. S., 2003, An outline of Celtiberian grammar, Friburg, ed. digital http ://www.freidok.unifreiburg.de 